UPR-723T, IASSNS-HEP-96/107, PUPT-1656

hep-th/9610238

October 1996

\title{
String and M-Theory Cosmological Solutions with Ramond Forms
}

\author{
André Lukas ${ }^{1 *}$, Burt A. Ovrut ${ }^{13}$ and Daniel Waldram ${ }^{2}$ \\ ${ }^{1}$ Department of Physics, University of Pennsylvania \\ Philadelphia, PA 19104-6396, USA \\ ${ }^{2}$ Department of Physics \\ Joseph Henry Laboratories, Princeton University \\ Princeton, NJ 08544, USA \\ ${ }^{3}$ School of Natural Sciences, Institute for Advanced Study \\ Olden Lane, Princeton, NJ 08540, USA
}

\begin{abstract}
A general framework for studying a large class of cosmological solutions of the low-energy limit of type II string theory and of M-theory, with non-trivial Ramond form fields excited, is presented. The framework is applicable to spacetimes decomposable into a set of flat or, more generally, maximally symmetric spatial subspaces, with multiple non-trivial form fields spanning one or more of the subspaces. It is shown that the corresponding low-energy equations of motion are equivalent to those describing a particle moving in a moduli space consisting of the scale factors of the subspaces together with the dilaton. The choice of which form fields are excited controls the potential term in the particle equations. Two classes of exact solutions are given, those corresponding to exciting only a single form and those with multiple forms excited which correspond to Toda theories. Although typically these solutions begin or end in a curvature singularity, there is a subclass with positive spatial curvature which appears to be singularity free. Elements of this class are directly related to certain black $p$-brane solutions.
\end{abstract}

*Supported by Deutsche Forschungsgemeinschaft (DFG) and Nato Collaborative Research Grant CRG. 940784. 


\section{Introduction}

An important constraint on string theory or any generalization of string theory, such as M-theory, is that it should be compatible with the standard model of early universe cosmology. The standard approach to string cosmology has been to consider an epoch when the evolution of the universe is described by the string low-energy effective action. One encounters a number of the familiar problems in matching this description to standard cosmology [1]. In particular, it is necessary to invoke some mechanism for stabilizing the massless string moduli such as the dilaton. While understanding these issues may in fact be central to understanding string cosmology [2], the usual approach has been at least to start by studying the simple cosmological solutions of the pure effective supergravity theory.

In the past, most focus has been on heterotic strings as the best model of low-energy particle physics. Consequently, study of cosmological solutions has concentrated on the dynamics of the dilaton and the compactified space in cosmological solutions of the effective theory. However, with the discovery of string dualities [3] and the existence of D-brane states [4], the nature of string theory has changed dramatically. Strong-weak coupling duality symmetries connect each of the five consistent supersymmetric string theories together with eleven-dimensional supergravity [5]. As a consequence, type II and eleven-dimensional supergravities may now be directly relevant to low-energy particle physics and cosmology [6]. Both theories contain form fields, namely a three form in eleven-dimensions and Ramond-Ramond (RR) forms of various degrees in type II theories. The importance of considering the excitation of these fields has been stressed by the discovery of objects carrying RR form-field charge, the D-branes of open string theory, and the central role these states have played in understanding the spacetime structure of black holes [7]. Given this change of perspective, it clearly becomes important to study the cosmological solutions of type II and eleven dimensional supergravity with non-trivial form fields excited. In a recent letter [8], we gave the first study of a class of such solutions, giving specific examples with one or more forms excited. The purpose of this paper is to provide a more detailed and complete description of this class of solutions.

The gravitational part of our Ansatz is based on the standard "rolling radii" solutions of Mueller [9], which are a generalization the classical Kasner solutions of Kaluza-Klein gravity [10]. In the original Mueller solutions spacetime is divided into a set of maximally symmetric spatial subspaces, each with its own time-dependent scale factor. (One subspace should be three-dimensional to describe our observed spacetime.) The rate of expansion or contraction of the subspaces is controlled by a power law and, typically, the universe either starts or ends in a curvature singularity. Including a dilaton in ten dimensions does not significantly alter the solution. This is not surprising given that one may consider the dilaton as the radius of a compact eleventh dimension. As was first pointed out in [11], in general such an evolution describes a motion on a moduli space with coordinates 
given by the values of the scale factors together with the dilaton. (We should point out that this is not quite the conventional notion of a string moduli space. For instance, it includes a scale factor "modulus" for our observed four-dimensional spacetime, and does not include all the possible moduli of, for example, toroidal compactification, since it assumes the torus is diagonal.)

We will adopt this rolling radii Ansatz for the metric and the dilaton. However, the point of this paper is to study solutions of type II theories and M-theory by turning on non-trivial RamondRamond form-fields. Can we understand how these will modify the moduli space description? Such solutions were first considered in the context of eleven-dimensional supergravity by Freund and Rubin [12]. Solutions with a non-trivial Neveu-Schwarz two-form field, usually in four dimensions, have also been discussed by various authors [13, 14, 15, 16, 17]. Following Freund [13], if we are to preserve the structure of a spacetime split into a set of maximally symmetric subspaces, the form fields must be chosen to span completely one or more of the subspaces. They may or may not in addition have a component in the time direction. Our central result will be to show that the effect of such a configuration is to introduce new exponential potential terms into the moduli space equations. In general the new equations describing the evolution of the dilaton and the scalefactors can only be solved exactly in certain special cases. We presented some examples of these special cases in a previous letter [8]. (One example appeared at almost the same time in paper by Kaloper [18].) A subsequent paper by Lü et al [19], gave a broad class of further exact solutions. Recently, cosmological solutions with Ramond forms obtained from black hole solutions have also been studied [16, 20]. The present paper provides a general framework for analyzing cosmologies with Ramond forms, the Neveu-Schwarz form and the cosmological constant of type II theories as well as those in eleven dimensions.

For all the cases we can solve exactly, our general result is that the moduli-space potential which arises when non-trivial form-fields are excited, significantly effects the structure of the solution. When all the subspaces are flat, the potential is operative over a particular, finite part of the evolution; at the extremes, the solution becomes pure geodesic motion and we return to the simple Kaluza-Klein Kasner-type solutions with some subspaces expanding, some contracting. (There is consequently always either an initial or a final curvature singularity.) Thus the effect of the form fields is to interpolate between two different Kaluza-Klein solutions. As we have mentioned, the expansion or contraction in the Kaluza-Klein solutions is controlled by a power law. In general, for solutions with an initial singularity, one finds that the rate of expansion is always sub-luminal and so there is no inflation. On the other hand, those solutions with a final singularity, just as in the pre-big-bang models [21], may exhibit superinflation but are unphysical as stands because the inflation ends in a curvature singularity.

The solution can be very different, however, when we allow for curved subspaces. The effect of the curvature is to introduce new terms into the moduli space potential, with the particular new feature that, in the case of a spherical subspace, the new term is always negative. This can 
significantly change the singularity structure, in some cases giving solutions where the curvature always remains finite. This suggest the interesting possibility that there may exist inflating solutions which are not forced to end in a singularity.

It is worth noting that there is actually a close connection between cosmological solutions and those describing $p$-branes, as was pointed out for the first time in ref. [B] and recently analyzed, starting from black hole solutions, in [20]. In the single $p$-brane solution [5], all the fields depend on the radial direction transverse to the brane. If the brane is elementary the form field has a component in the radial direction. If it is solitonic it spans a sphere around the brane, with no radial component. Since our solutions depend on time alone, time must play the role of the radial coordinate. Thus these solutions should correspond to purely spacelike $p$-branes, spatial $(p+1)$ dimensional objects which appear at one instant in time. Similarly, the two possible orientations of the form field in our solution, with a time component or without, correspond to the elementary and solitonic branes respectively. There is really one subtlety in this interpretation, at least when all the subspaces are flat. A standard $p$-brane solution in $D$ dimensions has a $(D-p-1)$-dimensional transverse space, composed of a radial direction and a $(D-p-2)$-sphere, whereas, in the cosmological solutions, time is the only transverse direction. However it is well known that the number of effective transverse directions can be reduced by stacking arrays of parallel $p$-branes together [22]. In the limit of smooth distribution of branes the fields no longer depend on the transverse coordinate along the direction of the array but only on the coordinates transverse to both the brane and the array. Thus we see that our cosmological solutions should really correspond to a distribution of parallel, spacelike branes evenly spread throughout space, though again appearing at only one instant in time. Solutions with multiple form fields excited then correspond to distributions of intersecting spacelike brane 23]. It is known that $p$-branes can be considered as interpolating solutions between different purely gravitational backgrounds. Therefore, the result that our cosmological solutions interpolate between pure Kaluza-Klein solutions supports the above analogy. The analogy breaks down however with regard to supersymmetry. Unlike the case of conventional $p$-brane solutions, we found no cosmological solutions which preserved any supersymmetries. We will see that the actual form of the solutions is closer to the black $p$-brane [24, 5] and other non-extremal brane solutions [25].

If one allows non-flat subspaces the $p$-brane analogy can become even clearer. Behrndt and Förste [16] and, more recently, Poppe and Schwager [20] have obtained cosmological solutions directly from black $p$-brane solutions by going to a regime where roles of the time and radial coordinate are exchanged. Such solutions correspond in our formalism to allowing a single spherical subspace. This subspace together with the time direction then constitute the space transverse to a single $p$-brane. This raises the possibility of finding new cosmological solutions of this form which correspond directly to single black $p$-brane states.

The organization of the paper is as follows. In section two we give the general structure of the 
solutions. Starting with the form of the effective action, we give the metric Ansatz and discuss the possible elementary- and solitonic-type Ansätze for the form fields. We then describe the action in moduli space which reproduces the spacetime equations of motion, and discuss its form. In section three, we give the exact general solution corresponding to exciting a single form field with flat subspaces, and discuss its structure as an interpolating solution between two pure Kaluza-Klein solutions, together with an explicit example. In section four, we give a class of exact solutions with multiple forms excited. These are solvable because they correspond to Toda models. Again, giving explicit examples in two distinct cases with flat subspaces, we discuss the interpolating form of these solutions. In section five, we explain how to incorporate the case of curved spatial subspaces into our formalism. A class of solutions appears to be related to black $p$-branes. We give, as an example, the solution of Behrndt and Förste [16], and discuss the possibility that those solutions which are related to black $p$-branes do not begin or end in a curvature singularity. We present some very brief concluding remarks in section six.

\section{General framework}

In this section we present a general framework for finding cosmological solutions with non-trivial form fields. While here the discussion will be as general as possible, we briefly discuss the same analysis in terms of simple, illustrative example in section 3.2. The starting point for our investigation is the following effective action

$$
\bar{S}=\int d^{D} x \sqrt{\bar{g}}\left[e^{-2 \phi}\left(\bar{R}+4(\partial \phi)^{2}-\frac{1}{12} H^{2}\right)-\sum_{r} \frac{1}{2\left(\delta_{r}+1\right) !} F_{r}^{2}-\Lambda\right]
$$

with the $D$-dimensional string frame metric $\bar{g}_{M N}$, the dilaton $\phi$, the NS 2-form $H$ and a number of RR $\delta_{r}$-forms $F_{r}=d A_{r}$. We also allow for a cosmological constant $\Lambda$ which appears in the massive extension of IIA supergravity [26]. Assuming this origin, it is restricted to be positive, $\Lambda>0$. Note also that this cosmological constant behaves much like a $\mathrm{RR}$ form in that is does not couple to the dilaton in the string frame. In this respect it is quite different from a "normal" cosmological constant appearing in an effective action of noncritical dimension which, like the NS field, couples to $\exp (-2 \phi)$.

The above action can account for a wide range of cosmological solutions in type II theories (where we usually have $D=10$ in mind) and, if the dilaton is set to zero, in $D=11$ supergravity. For simplicity we have kept only the kinetic terms for the form fields. In general, in both type II theories and eleven dimensional supergravity there are additional terms involving the coupling of form fields. We shall assume throughout this paper that for the configurations with which we are concerned, these terms do not contribute to the equations of motion. This will always be true if we only excite one orientation (in the sense given below) of a single form. It is not necessarily true when multiple forms are excited. 
In order to give a physical description of our solutions, we prefer to work in the canonical Einstein frame metric $g_{M N}$ which is related to the string frame metric by a conformal rescaling $g_{M N}=\exp (-4 /(D-2) \phi) \bar{g}_{M N}$. The corresponding action reads

$$
S=\int d^{D} x \sqrt{-g}\left[R-\frac{4}{D-2}(\partial \phi)^{2}-\sum_{r} \frac{1}{2\left(\delta_{r}+1\right) !} e^{-a\left(\delta_{r}\right) \phi} F_{r}^{2}-\Lambda e^{-a_{\Lambda} \phi}\right]
$$

where the NS field $H$ has been included in the sum over $F_{r}$. It is distinguished from the other forms by the dilaton couplings $a\left(\delta_{r}\right)$ given by

$$
a\left(\delta_{r}\right)=\left\{\begin{array}{ccc}
\frac{8}{D-2} & \mathrm{NS} & 2-\text { form } \\
\frac{4 \delta_{r}-2(D-2)}{D-2} & \mathrm{RR} & \delta_{r}-\text { form }
\end{array} .\right.
$$

The coupling $a_{\Lambda}$ for the cosmological constant

$$
a_{\Lambda}=-\frac{2 D}{D-2}
$$

equals the negative of the one for a $\mathrm{RR}(D-1)$-form. This reflects the fact that it couples to the 8-brane solution [27] of IIA supergravity.

The type of solutions we consider are characterized by a space split into $n$ flat subspaces, each of them characterized by a scale factor $\alpha_{i}$. We concentrate on flat subspaces since this covers already a wide range of solutions and seems to be appropriate for our main purpose, namely to investigate the effect of the forms. The effect of curved maximally symmetric subspaces can, however, be easily incorporated into the formalism to be developed in this section. We will discuss this in section six. In the flat case, the corresponding metric is given by

$$
d s^{2}=-N^{2}(\tau) d \tau^{2}+\sum_{i=0}^{n-1} e^{2 \alpha_{i}(\tau)} d \mathbf{x}_{i}^{2}
$$

where $d \mathbf{x}_{i}^{2}$ is the measure of a $d_{i}$-dimensional flat maximally symmetric subspace and $\sum_{i=0}^{n-1} d_{i}=$ $D-1$. For solutions with this structure, the dilaton should depend on time only, $\phi=\phi(\tau)$. This Kaluza-Klein-type Ansatz is about the simplest allowing for the cosmologically key properties of homogeneity and isotropy as well as for an "external" and "internal" space.

For a ten dimensional theory, the simplest choice is to split up the space into two subspaces $(n=2)$ with $d_{0}=3$ and $d_{1}=6$. Then the $d_{0}=3$ part could be interpreted as the spatial part of "our" 4-dimensional space-time with an evolution described by the scale factor $\alpha_{0}$. The other six dimensions would form an internal space with a modulus $\alpha_{1}$. Clearly, one is free to split these six dimensions even further and to consider, for example, a situation with $n=3$ and $\left(d_{0}, d_{1}, d_{2}\right)=(3,4,2)$. From the cosmological point of view one might even allow for a further split of the 3 -dimensional space such as $\left(d_{0}, d_{1}, d_{2}\right)=(1,2,6)$ as long as the $1+2$-dimensional space approaches a 3 -dimensional isotropic space at late times. 
As discussed in the introduction, this Kaluza-Klein-type Ansatz is closely related to the form of $p$-brane solutions in supergravity theories. Consider the simple case of two subspaces with $d_{0}=p+1$ and $d_{1}=D-p-2$. For the standard $p$-brane solution, spacetime is split into a radial direction, transverse to the brane, upon which all the fields depend, a flat subspace describing the $(p+1)$-dimensional worldvolume of the brane and hence including a timelike direction, and a spherical subspace surrounding the brane. In our cosmological solution the fields depend only on the timelike direction and so time is the analog of the radial coordinate in the $p$-brane solutions. The $d_{0}$ subspace then becomes the analog of the brane worldvolume, though it is now completely spacelike, while the $d_{1}$ subspace becomes the sphere surrounding the brane. As described in the introduction, since we usually take the $d_{1}$ space to be flat rather than spherical, probably a more exact analogy is with a distribution of spacelike $p$-branes, rather than a single brane. More complicated cases, where the space is split into a number of subspaces, are analogous to multiple intersecting $p$-brane solutions [23].

The choice of subspaces is important in fixing the possible structure of the antisymmetric tensors fields. The symmetry of the above metric allows for two different Ansätze for the form fields which we call "elementary" and "solitonic" in analogy to the two types of $p$-brane solutions. They are characterized by the following nonvanishing components of the field strength.

- elementary : if $\sum_{i} d_{i}=\delta_{r}$ for some of the spatial subspaces $i$ we may set

$$
\left(F_{r}\right)_{0 \mu_{1} \ldots \mu_{\delta_{r}}}=A_{r}(\alpha) f_{r}^{\prime}(\tau) \epsilon_{\mu_{1} \ldots \mu_{\delta_{r}}}, \quad A_{r}(\alpha)=e^{-2 \sum_{i} d_{i} \alpha_{i}}
$$

where $\mu_{1} \ldots \mu_{\delta_{r}}$ refer to the coordinates of these subspaces, $f_{r}(\tau)$ is an arbitrary function to be fixed by the form field equation of motion, and the prime denotes the derivative with respect to $\tau$. With raised indices, the symbol $\epsilon^{\mu_{1} \ldots \mu_{\delta_{r}}}$ takes the values 0 or 1 and is completely antisymmetric on all $\delta_{r}$ indices. Note that the sum over $i$ in the exponent runs only over those subspaces which are spanned by the form.

An elementary form can therefore extend over one or more of the subspaces only if its degree matches the total dimension of these spaces. Consider for example the RR three-form of type IIA supergravity. If the space is split up as $\left(d_{0}, d_{1}\right)=(3,6)$ it fits into the 3 -dimensional subspace and the above general Ansatz specializes to $F_{0 \mu_{1} \mu_{2} \mu_{3}}=\exp \left(-6 \alpha_{0}\right) f^{\prime}(\tau) \epsilon_{\mu_{1} \mu_{2} \mu_{3}}$ where $\mu_{1}, \mu_{2}, \mu_{3}$ refer to the coordinates of this subspace. Taking $\left(d_{0}, d_{1}, d_{2}\right)=(1,2,6)$ allows for a similar Ansatz but the form now extends over the $1+2$-dimensional space and the exponential in the Ansatz changes to $\exp \left(-2 \alpha_{0}-4 \alpha_{1}\right)$.

- solitonic : if $\sum_{i} d_{i}=\delta_{r}+1$ for some of the spatial subspaces $i$ we may allow for

$$
\left(F_{r}\right)_{\mu_{1} \ldots \mu_{\delta_{r}+1}}=B_{r}(\alpha) w_{r} \epsilon_{\mu_{1} \ldots \mu_{\delta_{r}+1}}, \quad B_{r}(\alpha)=e^{-2 \sum_{i} d_{i} \alpha_{i}}
$$


where $\mu_{1} \ldots \mu_{\delta_{r}+1}$ refer to the coordinates of these subspaces and $w_{r}$ is an arbitrary constant. As for the elementary Ansatz, the sum over $i$ in the exponent runs over the subspaces spanned by the form. It is easy to check that this Ansatz, already solves the form equation of motion.

Note that in contrast to the elementary Ansatz, the solitonic field strength does not have a time index. Therefore the matching condition for the dimensions differs. Given, for example, a split $\left(d_{0}, d_{1}\right)=(3,6)$ one has to use a 2 form instead of a three-form to fit into the 3 -dimensional subspace. The above Ansatz then reads $F_{\mu_{1} \mu_{2} \mu_{3}}=\exp \left(-6 \alpha_{0}\right) w \epsilon_{\mu_{1} \mu_{2} \mu_{3}}$.

Why did we use the terms "elementary" and "solitonic" for the above Ansätze? This is again motivated by a close analogy to $p$-brane solutions. Consider again the simple split into two subspaces, $d_{0}=p+1$ and $d_{1}=D-p-2$, where $d_{0}$ is the analog of the $p$-brane worldvolume, and $d_{1}$ is the analog of the sphere surrounding the brane. There are two types of the simple brane solutions. Each is a source of a form field, but the field is aligned differently in the two cases. For the "electrically charged" elementary brane, the form field is aligned in the hyperplane of the brane together with the radial direction. This has an analog in our elementary Ansatz with the form aligned in the $d_{0}$ subspace together with the time direction. For the "magnetically charged" solitonic brane, the form field is aligned in the sphere surrounding the brane, which in turn has an analog in our solitonic Ansatz with the form aligned simply in the $d_{1}$ subspace.

Having specified the form of our Ansatz, we now look to solve the equations of motion derived from the action (2). However, it is in fact easy to show that, under a very mild restriction on the structure of forms to be discussed below, the resulting equations of motion can be obtained from a reduced Lagrangian which depends only on $\alpha_{i}, \phi, N$ and $f_{r}$, each as functions of $\tau$. The Lagrangian is given by

$$
\mathcal{L}=E\left[\sum_{i=0}^{n-1} d_{i} \alpha_{i}^{\prime 2}-\sum_{i, j=0}^{n-1} d_{i} d_{j} \alpha_{i}^{\prime} \alpha_{j}^{\prime}+\frac{4}{D-2} \phi^{\prime 2}+V_{e}-N^{2} V_{s}\right]
$$

with

$$
\begin{aligned}
V_{e} & =\frac{1}{2} \sum_{r} A_{r}(\alpha) e^{-a\left(\delta_{r}\right) \phi} f_{r}^{\prime 2} \\
V_{s} & =\frac{1}{2} \sum_{r} B_{r}(\alpha) w_{r}^{2} e^{-a\left(\delta_{r}\right) \phi}+\Lambda e^{-a_{\Lambda} \phi} \\
E & =\frac{1}{N} e^{\sum_{i=0}^{n-1} d_{i} \alpha_{i}}
\end{aligned}
$$

In the definitions of the potentials $V_{e}$ and $V_{s}$, the sum over $r$ is understood to run over all the elementary and solitonic configurations which have been chosen according to the given rules. In general, this even includes the possibility of having a superposition of two of these configurations for one form. However, in this case the nonvanishing components of the two configurations should differ in at least two spatial directions in order to obtain the correct equations of motion from 
Lagrangian (8) (otherwise the equations of motion contain an additional cross term which cannot be derived from eq. (8)). We will adopt this fairly mild restriction on the structure of the forms thereby gaining the advantage of having a simple description of our models in terms of a reduced Lagrangian.

The equations of motion for the functions $f_{r}$ to be derived from eq. (8) read

$$
\frac{d}{d \tau}\left(E A_{r} e^{-a\left(\delta_{r}\right) \phi} f_{r}^{\prime}\right)=0
$$

The first integrals are

$$
f_{r}^{\prime}=v_{r} E^{-1} A_{r}^{-1} e^{a\left(\delta_{r}\right) \phi}
$$

where $v_{r}$ are integration constants. Equation (11) can be used to eliminate $f_{r}^{\prime}$ from the elementary potential $V_{e}$. This then reduces the problem to solving the remaining equations of motion now given purely in terms of $\alpha_{i}, \phi$ and $N$.

In fact we find that the remaining equations can also be derived from a simple reduced Lagrangian. This Lagrangian describes the motion of a particle in a "moduli space" the coordinates of which are the scale factors together with the dilaton. We find $E$ is then the metric for the particle worldline in the moduli space. To see this structure, we first introduce the notation $\boldsymbol{\alpha}=\left(\alpha_{I}\right)=\left(\alpha_{i}, \phi\right)$ for a general point in the moduli space. We also define a particular metric on the moduli space $G_{I J}$ by

$$
\begin{aligned}
G_{i j} & =2\left(d_{i} \delta_{i j}-d_{i} d_{j}\right) \\
G_{i n} & =G_{n i}=0 \\
G_{n n} & =\frac{8}{D-2} .
\end{aligned}
$$

The equations of motion for $\boldsymbol{\alpha}$ and $N$ following from eq. (8) then take the simple form

$$
\begin{aligned}
\frac{d}{d \tau}\left(E G \boldsymbol{\alpha}^{\prime}\right)+E^{-1} \frac{\partial U}{\partial \boldsymbol{\alpha}} & =0 \\
\frac{1}{2} E \boldsymbol{\alpha}^{\prime T} G \boldsymbol{\alpha}^{\prime}+E^{-1} U & =0 .
\end{aligned}
$$

The quantity $U$ is given by

$$
U=e^{2 \sum_{i=0}^{n-1} d_{i} \alpha_{i}}\left(\frac{1}{N^{2}} V_{e}+V_{s}\right)
$$

where $f_{r}^{\prime}$ in $V_{e}$ has been replaced using eq. (11). Clearly, these equations of motion can be derived from the simple Lagrangian (it is convenient to make the change of variables from $N$ to $E$ ),

$$
\mathcal{L}=\frac{1}{2} E \boldsymbol{\alpha}^{\prime T} G \boldsymbol{\alpha}^{\prime}-E^{-1} U
$$

We note from its definition (12), that the metric on the moduli space has Minkowskian signature $(-++\cdots+)$, thus we are really free to interpret (16) as the worldline Lagrangian for particle moving in the moduli space. The first term is kinetic, while $U$ defines a potential in the moduli 
space. Further, $E$ is the metric on the particle worldline. It encodes the gauge freedom that we have not yet chosen a particular time parameterization to describe the evolution of the spacetime. As usual its equation of motion (14) is a constraint.

It is useful to rewrite the potential $U$ in a more systematic way as

$$
U=\frac{1}{2} \sum_{r=1}^{m} u_{r}^{2} \exp \left(\mathbf{q}_{r} \cdot \boldsymbol{\alpha}\right)
$$

where the sum runs over all elementary and solitonic configurations as well as a possible cosmological constant term. The constants $u_{r}$ represent the integration constants $v_{r}$ in eq. (11) for elementary forms, the constants $w_{r}$ in the Ansatz (7) for solitonic forms or a cosmological constant. The type of each term is specified by the vectors $\mathbf{q}_{r}$ which can be read off from eqs. (6), (7), (9) and the definition (15). For an elementary $\delta$-form they are given by

$$
\mathbf{q}^{(\mathrm{el})}=\left(2 \epsilon_{i} d_{i}, a(\delta)\right), \quad \epsilon_{i}=0,1, \quad \delta=\sum_{i=0}^{n-1} \epsilon_{i} d_{i}
$$

with $\epsilon_{i}=1$ if the form is nonvanishing in the subspace $i$ and $\epsilon_{i}=0$ otherwise. For type II theories the dilaton couplings $a(\delta)$ are given in eq. (3) and (4). To account for the $D=11$ case (or constant dilaton solutions) we may just set $a(\delta)=0$.

Let us give an example at this point. An elementary IIA RR 3 form, put into the first subspace of a $D=10$ space split with $\left(d_{0}, d_{1}\right)=(3,6)$, is characterized by a vector $\mathbf{q}=(6,0,-1 / 2)$. It generates a potential term in (17) which depends on $\alpha_{0}$ and the dilaton but not on $\alpha_{1}$. More generally, a potential term describing the effect of an elementary form depends only on those scale factors which correspond to subspaces spanned by the form. Since the entries $q_{i}$ of $\mathbf{q}$ are always positive, the potential tends to drive the scale factors for these subspaces to smaller values. Therefore these subspaces tend to be contracting or at least less generically expanding than others.

The situation for a solitonic $\delta$-form is in some sense complementary. It is specified by a vector

$$
\mathbf{q}^{(\mathrm{sol})}=\left(2 \tilde{\epsilon}_{i} d_{i},-a(\delta)\right), \quad \tilde{\epsilon}_{i} \equiv 1-\epsilon_{i}=0,1, \quad \tilde{\delta} \equiv D-2-\delta=\sum_{i=0}^{n-1} \tilde{\epsilon}_{i} d_{i}
$$

with $\tilde{\epsilon}_{i}=1$ if the form vanishes in the subspace $i$ and $\tilde{\epsilon}_{i}=0$ otherwise. For example, a solitonic IIB RR 2 form in the first subspace of a space split as $\left(d_{0}, d_{1}\right)=(3,6)$ is specified by $\mathbf{q}=(0,12,1)$. It generates a potential term in (17) which depends on $\alpha_{1}$ and the dilaton but not on $\alpha_{0}$. More generally, in contrast to the elementary case, the potential term now depends on those scale factors corresponding to subspaces not spanned by the form.

Finally, a cosmological constant is characterized by

$$
\mathbf{q}^{(\Lambda)}=\left(2 d_{i}, \frac{2 D}{D-2}\right) \text {. }
$$

Note that for all these vectors $\sum_{i=0}^{n-1} q_{i}>0$, a fact which we will use later on. The moduli space metric allows us to define a natural scalar product on the space of vectors q.. It is the product 
$\mathbf{q} \cdot \boldsymbol{\alpha}$ that appears in the potential $U$. Thus $\mathbf{q}$ is naturally a covariant rather than a contravariant vector in the moduli space, so we define

$$
<\mathbf{q}_{1}, \mathbf{q}_{2}>=\mathbf{q}_{1}^{T} G^{-1} \mathbf{q}_{2}
$$

with the inverse of $G$ given by

$$
\begin{aligned}
\left(G^{-1}\right)_{i j} & =-\frac{1}{2(D-2)}+\frac{1}{2 d_{i}} \delta_{i j} \\
\left(G^{-1}\right)_{i n} & =\left(G^{-1}\right)_{n i}=0 \\
\left(G^{-1}\right)_{n n} & =\frac{D-2}{8} .
\end{aligned}
$$

Since the metric $G$ has Minkowskian signature, we can distinguish between space- and time-like vectors q. As we will see, the structure of the solutions depends crucially on this distinction.

Generically, the models specified by the eqs. (13), (14) and (17) cannot be solved. A complete solution, however, can be found if the potential $U$ consists of one exponential term only or if contact with Toda theory can be made. These two cases will be discussed in section 3 and 4 . An interesting observation at this stage is that the potential $U$ may contain exponentials with different signs in front of the dilaton $\phi$ and therefore may have dilaton minima at finite values. It is an important question how this influences the dynamics of the dilaton, in particular since the stabilization of the dilaton is one of the major problems of string cosmology. This will be analyzed more completely elsewhere [28].

\section{Solutions with one potential term}

In this section, we will analyze models with just one form turned on (or a non-zero cosmological constant). The form may be elementary or fundamental and there may be any number of subspaces. All of these cases correspond to a potential

$$
U=\frac{1}{2} u^{2} \exp (\mathbf{q} \cdot \boldsymbol{\alpha})
$$

where $u^{2}>0$. Here $\mathbf{q}$ is one of the vectors specified at the end of the last section. We will start by giving the general form of the solution and then give a simple example in section 3.2.

One way of solving the equations of motion for a potential (23) is to use the gauge freedom in the definition of the time coordinate. We can always choose a gauge such that

$$
N=\exp ((\mathbf{d}-\mathbf{q}) \cdot \boldsymbol{\alpha})
$$

where we introduce a vector giving the subspace dimensionsd $=\left(d_{i}, 0\right)$. This implies $E=\exp (\mathbf{q} \cdot \boldsymbol{\alpha})$ and the following set of equations for $\boldsymbol{\alpha}$

$$
\frac{d}{d \tau}\left(G E \boldsymbol{\alpha}^{\prime}\right)+\frac{1}{2} u^{2} \mathbf{q}=0
$$




$$
\frac{E}{2} \boldsymbol{\alpha}^{T^{\prime}} G \boldsymbol{\alpha}^{\prime}+\frac{1}{2} u^{2}=0
$$

In this form they can be integrated immediately, leading to the general solution

$$
\boldsymbol{\alpha}=\mathbf{c} \ln \left|\tau_{1}-\tau\right|+\mathbf{w} \ln \left(\frac{s \tau}{\tau_{1}-\tau}\right)+\mathbf{k}
$$

where

$$
\mathbf{c}=\frac{2 G^{-1} \mathbf{q}}{<\mathbf{q}, \mathbf{q}>}
$$

The sign $s= \pm 1$ is determined by $s=\operatorname{sign}(<\mathbf{q}, \mathbf{q}>)$ 円 and $\mathbf{w}, \mathbf{k}$ are integration constants subject to the constraints

$$
\begin{aligned}
\mathbf{q} \cdot \mathbf{w} & =1 \\
\mathbf{w}^{T} G \mathbf{w} & =0 \\
\mathbf{q} \cdot \mathbf{k} & =\ln \left(\frac{u^{2} \mid<\mathbf{q}, \mathbf{q}>1}{4}\right) .
\end{aligned}
$$

$\tau_{1}$ is a free parameter which we can take to be positive. The opposite choice $\tau_{1}<0$ leads to a shift in the range of $\tau$ which does not affect the physics of the solution. The range of $\tau$ should be chosen to ensure a positive argument of the second logarithm in eq. (26). This depends on the sign of $\langle\mathbf{q}, \mathbf{q}\rangle$ and we have the two cases

$$
\begin{array}{cc}
0<\tau<\tau_{1} & \text { for } \quad<\mathbf{q}, \mathbf{q}>>0 \\
\tau<0 \text { or } \tau>\tau_{1} & \text { for } \quad<\mathbf{q}, \mathbf{q}><0
\end{array} .
$$

Which of these cases is actually realized in type II models? Using the vectors q given in the end of section 2 , we find for a solitonic or elementary $\delta$ form (or a cosmological constant which is similar to a $\mathrm{RR}(D-1)$-form $)$

$$
<\mathbf{q}, \mathbf{q}>=\frac{D-2}{8} a(\delta)^{2}+\frac{2}{D-2} \delta \tilde{\delta}=\left\{\begin{array}{cl}
4 & \mathrm{NS} \\
\frac{D-2}{2} & \mathrm{R}
\end{array}\right.
$$

which is always positive. Also the $D=113$ form leads to a positive result, as can be seen from the above formula by setting $a(\delta)=0$. We conclude that, in the present context, we are dealing with spacelike vectors $\mathbf{q}$ only, and we have $0<\tau<\tau_{1}$.

For concreteness, let us give an example of how to compute the coefficients $\mathbf{c}$ for a given model. Consider an elementary $\delta$ form in $D=10$ and a spacetime split into two subspaces with dimensions $d_{0}=\delta$ and $d_{1}=D-\delta-1=\tilde{\delta}+1$. Then from eq. (12) we find the metric

$$
G=\left(\begin{array}{ccc}
-2 \delta(\delta-1) & -2 \delta(\tilde{\delta}+1) & 0 \\
-2 \delta(\tilde{\delta}+1) & -2 \tilde{\delta}(\tilde{\delta}+1) & 0 \\
0 & 0 & 1
\end{array}\right)
$$

\footnotetext{
${ }^{1}$ Here we disregarded the somewhat marginal possibility that $\mathbf{q}$ is a null vector, i. e. $<\mathbf{q}, \mathbf{q}>=0$.
} 
and from eq. (22) its inverse

$$
G^{-1}=\left(\begin{array}{ccc}
\frac{1}{2 \delta}-\frac{1}{16} & -\frac{1}{16} & 0 \\
-\frac{1}{16} & \frac{1}{2(\tilde{\delta}+1)}-\frac{1}{16} & 0 \\
0 & 0 & 1
\end{array}\right) .
$$

Inserting this into eq. (27), together with the vector $\mathbf{q}=(2 \delta, 0, a(\delta))$ characterizing the elementary $\delta$ form, we find the following coefficient $\mathbf{c}$ of the first term in the solution (26)

$$
\mathbf{c}=\left(\frac{\tilde{\delta}}{2(\delta+\tilde{\delta})},-\frac{\delta}{2(\delta+\tilde{\delta})}, \frac{a(\delta)}{2}\right) .
$$

The above example provides useful insight into the relation of our solutions to $p$-brane solutions. As discussed in the last section, we would expect this example to correspond to an elementary $(\delta-1)$ brane solution. Eq. (26) has been written in a form which makes this relation transparent. The first term in eq. (26) is the analog of an extremal, BPS, p-brane solution. To see this, we remind the reader that a brane BPS solutions is characterized by a proportionality of all $\alpha_{I}$ to a certain harmonic function $h$; that is $\alpha_{I} \sim h$. The $(\delta-1)$-brane constants of proportionality [5] are exactly reproduced by eq. (33). The second term in eq. (26) represents the analog of nonextremal extensions which also have been studied in the $p$-brane case [24, 5, 25]. The difference here is that the second term cannot be set to zero since $w_{I}=0$ for all $I$ is incompatible with the constraints (28). Another possibility is to choose $\tau_{1}=0$ which by eq. (29) works for timelike vectors q only. Unfortunately, as we saw, such vectors do not occur within $D=10$ type II and $D=11$ supergravity. By analogy with $p$-brane solutions one would therefore conclude that the solution (26) does not preserve any of the supersymmetries of these theories. This can, in fact, be checked explicitly for certain examples by using the supersymmetry transformations of the gravitino.

So far, our solutions have been expressed in terms of the time parameter $\tau$ which is defined by the gauge choice (24) for $N(\tau)$. For a discussion of the cosmological properties of our models, however, we should reexpress them in terms of the comoving time $t$, that is in the gauge where the $N=1$. This can be done by integrating the defining relation $d t=N(\tau) d \tau$. The explicit expression for $N(\tau)$ is given by inserting the solution (26) into the gauge fixing equation (24) for $N(\tau)$, which gives

$$
N=\left.\exp ((\mathbf{d}-\mathbf{q}) \cdot \mathbf{k})\left|\tau_{1}-\tau\right|^{-x+\Delta-1}|| \tau\right|^{x-1}
$$

with

$$
\begin{aligned}
x & =\mathbf{d} \cdot \mathbf{w} \\
\Delta & =\mathbf{d} \cdot \mathbf{c}=2 \frac{\langle\mathbf{d}, \mathbf{q}\rangle}{\langle\mathbf{q}, \mathbf{q}\rangle} .
\end{aligned}
$$


Note that the quantity $x$ depends on the specific choice of integration constants $\mathbf{w}$ whereas $\Delta$ is a fixed number for a given model (once the dimensions $\mathbf{d}$ of the subspaces and the Ansatz for the form encoded in $\mathbf{q}$ have been chosen). Depending on the values of $x$ and $\Delta$, the gauge parameter $N$ may have singularities at $\tau=0$ and $\tau=\tau_{1}$. This determines the allowed range in the comoving time $t$ as we will discuss in detail in the next subsection.

Another quantity which is of importance in discussing the physical content of our solutions is the scalar curvature $R$. For the solution (26) it is given by

$$
R \sim\left|\tau_{1}-\tau\right|^{2(x-\Delta)}|\tau|^{-2 x} P_{2}(\mathbf{w}, \boldsymbol{\alpha}, \tau)
$$

where $P_{2}$ is a second order polynomial in $\tau$ which we will not need explicitly. We have also omitted an unimportant constant of proportionality which depends on the integration constants. The first two factors in this equation indicate potential singularities at $\tau=0$ and $\tau=\tau_{1}$, depending on $x$ and $\Delta$ as in the case of the gauge parameter $N$. However, in contrast to singularities in $N$, such singularities are true coordinate independent curvature singularities. They will be further discussed in the next subsection.

\subsection{Cosmology of solutions with spacelike q-vectors}

As already mentioned, the case of spacelike q-vectors is the most important in our context since all vectors $\mathbf{q}$ appearing within the $D=10$ type II theories and $D=11$ supergravity are spacelike. Even under this restriction on $\mathbf{q}$, our solution (26) covers a large number of different models. In this section, we will discuss the cosmological properties which can be extracted from these models in general. A concrete illustrating example will be given in the next subsection.

Recall that the singularity structure of the solution (26) is determined by the quantities $x$ and $\Delta$ defined in eq. (35) and (36). What values are actually allowed for these quantities? From $<\mathbf{d}, \mathbf{q}>=-\sum_{j=0}^{n-1} q_{j} / 2(D-2)$ and $\sum_{j=0}^{n-1} q_{j}>0$ it follows from eq. (36) that $\Delta<0$. Though we are not able to give a general proof, the parameter $x$, which unlike $\Delta$ depends on the parameters of the solution, turns out to be either $x<\Delta$ or $x>0$ in all specific examples we considered. This divides the set of initial conditions into two disconnected subsets corresponding to two classes of solutions with different properties.

We begin our discussion of these properties by analyzing the allowed ranges in comoving time $t$. Recall from eq. (29) that the time parameter $\tau$ is always in the range $0<\tau<\tau_{1}$ since we have $<\mathbf{q}, \mathbf{q}>>0$. The singularity structure of the gauge parameter $N$ in eq. (34) then shows that this range is mapped to the following ranges in $t$

$$
\tau \rightarrow t \in\left\{\begin{array}{lll}
{\left[-\infty, t_{1}\right]} & \text { for } x<\Delta<0, & (-) \text { branch } \\
{\left[t_{0},+\infty\right]} & \text { for } x>0>\Delta, & (+) \text { branch }
\end{array} .\right.
$$


Here $t_{0}$ and $t_{1}$ are two finite unrelated values that appear as integration constants from integrating $d t=N(\tau) d \tau$. Thus we have found two disconnected branches corresponding to asymptotically positive and negative time ranges.

Let us next discuss the scalar curvature in each branch. We start with the $(-)$ branch. As inspection of eq. (37) shows, the curvature vanishes as $t \rightarrow-\infty(\tau \simeq 0)$ since $x<\Delta<0$. With increasing time $R$ grows and, finally, the system runs into the curvature singularity at $t=t_{1}$ $\left(\tau=\tau_{1}\right)$ since the power $2(x-\Delta)$ of the first term in eq. (37) is negative. Therefore, classically the $(-)$ solution cannot be continued beyond this point.

In the $(+)$ branch the situation is similar but reversed in time. At $t=t_{0}(\tau=0)$ we find a singularity since $x>0$ in this branch. The solution cannot be extended into the past. As $t \rightarrow \infty$ $\left(\tau \simeq \tau_{1}\right)$ the curvature behaves smoothly and approaches zero.

Though generically correct, the above argument has a loophole. For very specific values of the initial parameters $\mathbf{w}$, the polynomial $P_{2}$ is proportional to $\tau$ or $\tau_{1}-\tau$ so that it can cancel against one of the first two factors in eq. (37) which cause the singularity. If $|x|$ is sufficiently small, the singularity may disappear completely. For the $(-)$ branch this is realized if $w_{n}=c_{n}$ and $x \geq-1 / 2$. For the $(+)$ branch it occurs if $w_{n}=0$ and $x \leq 1 / 2$. This phenomenon is quite similar to what happens in the curvature singularity free WZW model of ref. 29]

So far, we have considered quantities which provide information about the behaviour of the total $D$ dimensional space only. Let us now turn to the individual subspaces of dimension $d_{i}$. To analyze their behaviour, we should calculate their respective Hubble parameters $H_{i}$ in terms of the comoving time. In fact, it is possible to explicitly express the comoving time $t(\tau)$ in terms of hypergeometric functions. It is, however, more instructive to look at the asymptotic regions $\tau \simeq 0$ (corresponding to $t \rightarrow-\infty$ for the $(-)$ branch and $t \simeq t_{0}$ for the $(+)$ branch) and $\tau \simeq \tau_{1}$ (corresponding to $t \simeq t_{1}$ for the $(-)$ branch and $t \rightarrow \infty$ for the $(+)$ branch). In these regions the Hubble parameters can be written as $\mathrm{Z}$

$$
\mathbf{H} \equiv \dot{\boldsymbol{\alpha}}=\frac{\mathbf{p}}{t-t_{s}}
$$

with the constant expansion coefficients $\mathbf{p}$ satisfying

$$
\mathbf{p} G \mathbf{p}=0, \quad \mathbf{d} \cdot \mathbf{p}=1
$$

The time shift $t_{s}$ depends on the asymptotic region and branch under consideration. The sign of $t-t_{s}$, however, is always well defined : It is negative in the $(-)$ branch and positive in the $(+)$ branch. If we combine the two equations (40) and use the explicit form of the metric $G$ in (12) we find

$$
\frac{4}{D-2} p_{\phi}^{2}+\sum_{i=0}^{n-1} d_{i} p_{i}^{2}=1 .
$$

\footnotetext{
${ }^{2}$ The dot denotes the derivative with respect to the comoving time $t$.
} 
The explicit expressions for $\mathbf{p}$ in terms of the integration constants are

$$
\mathbf{p}=\left\{\begin{array}{cll}
\frac{\mathbf{w}}{x} & \text { at } & \tau \simeq 0 \\
\frac{\mathbf{w}-\mathbf{c}}{x-\Delta} & \text { at } & \tau \simeq \tau_{1}
\end{array} .\right.
$$

They have been calculated using the general solution (26) and the asymptotic limits of $N(\tau)$ to be read off from eq. (34). The behavior of the Hubble parameters (39) along with eq. (40) indicates that the solutions behave asymptotically like those of pure Kaluza-Klein theory with a dilaton. This can be seen by a comparison with the solutions of ref. [9]. Therefore, one expects that the potential $U$ provided by the form is effectively turned off in these limits. In fact, inserting the general solution (26) into the potential (23), we find $U \sim\left(\tau_{1}-\tau\right) \tau$ which implies that $U$ is effectively zero near $\tau \simeq 0$ and $\tau \simeq \tau_{1}$. The effect of the form is therefore to generate a mapping $\mathbf{p}(\tau \simeq 0) \rightarrow \mathbf{p}\left(\tau \simeq \tau_{1}\right)$ between two pure Kaluza-Klein states.

What do the above results mean for the evolution of the subspaces? We consider the $(+)$ branch first. Remember that $t-t_{s}>0$ in this branch so that from eq. (39) a positive $p_{i}$ results in expansion and a negative $p_{i}$ in contraction. Moreover, the equation $\mathbf{d} \cdot \mathbf{p}=1$ shows that at least one of the $p_{i}$ has to be positive. Consequently, at least one of the subspaces has to be expanding. From eq. (41) we conclude that $\left|p_{i}\right|<1$ always. The expansion is therefore subluminal, i. e. the scale factor $e^{\alpha_{i}}$ grows less quickly than the horizon size $H_{i}^{-1}$. This behaviour is similar to a radiation or matter dominated universe corresponding to $p_{i}=1 / 2$ and $p_{i}=2 / 3$, respectively.

The situation is completely different in the $(-)$ branch. Since $t-t_{s}<0$, a positive $p_{i}$ results in contraction and a negative $p_{i}$ in expansion. Now we conclude from $\mathbf{d} \cdot \mathbf{p}=1$ that at least one subspace must be contracting. Since we are in the negative time range, eq. (39) shows that expansion $\left(H_{i}>0\right)$ goes along with an increasing $H_{i}$, that is, a shrinking horizon size. Such a behaviour is also called superinflation since scales are stretched across the horizon even more rapidly than in "ordinary" inflation where the horizon size is approximately constant. It is very similar to the superinflating expansion in pre-big-bang models [21]. However, it should be stressed that it was obtained in the Einstein frame as opposed to the string frame used in those models.

Our solutions allow various patterns of expanding and contracting spatial subspaces. The details of the evolution depend on the partition $\left\{d_{i}\right\}$, the form and the subspaces it occupies and the initial conditions. Examples with 3 expanding and 6 contracting spatial dimensions as $t \rightarrow \infty$ exist, as were given in ref. [8]. Though this is by no means preferred in the present context, its existence is not entirely trivial since some models fail to reproduce such a "desired" situation. The effect of the form can be quite dramatic. For example, it can reverse expansion and contraction of two subspaces during the early asymptotic period into its converse during the late period.

A "preferred" cosmological scenario suggested by these solutions consists of a combination of the $(-)$ and the $(+)$ branch to account for inflation as well as for a postinflationary subluminal expansion. It is remarkable that such a pre-big-bang scenario can be realized in the Einstein 
frame. The apparent shortcoming of this scenario is that the $(-)$ and the $(+)$ branch constitute two different a priori unrelated solutions. As for string frame pre-big-bang models, one might argue [21] that scale factor ( $\mathrm{T}$ ) duality between the branches provides the correct transition and some hints for this have been found in a two dimensional toy model [30].

\subsection{An example}

Up to this point our discussion has been rather general. Let us now illustrate the steps in our solution by giving a simple example. It is not meant to represent the most realistic case in the cosmological sense, but rather to explain our general results as concretely as possible.

We consider the following situation : 10-dimensional spacetime is split into two subspaces with $\mathbf{d}=\left(d_{0}, d_{1}, 0\right)=(3,6,0)$ and an elementary IIA RR 3 form occupies the 3 -dimensional subspace. This implies the Ansatz

$$
\begin{aligned}
d s^{2} & =-N^{2}(\tau) d \tau^{2}+e^{2 \alpha_{0}} d \mathbf{x}_{0}^{2}+e^{2 \alpha_{1}} d \mathbf{x}_{1}^{2} \\
F_{0 \mu_{1} \mu_{2} \mu_{3}} & =e^{-6 \alpha_{0}} f^{\prime}(\tau) \epsilon_{\mu_{1} \mu_{2} \mu_{3}} \\
\phi & =\phi(\tau)
\end{aligned}
$$

in accordance with the eqs. (5), (6). The equations of motion for this example can be derived from the Lagrangian

$$
\mathcal{L}=E\left[-6 \alpha_{0}^{\prime 2}-30 \alpha_{1}^{\prime 2}-36 \alpha_{0}^{\prime} \alpha_{1}^{\prime}+\frac{1}{2}{\phi^{\prime}}^{2}+V_{e}\right]
$$

with the elementary potential $V_{e}$ and $E$ given by

$$
V_{e}=\frac{1}{2} e^{-6 \alpha_{0}+\frac{1}{2} \phi} f^{\prime 2}, \quad E=\frac{1}{N} e^{3 \alpha_{0}+6 \alpha_{1}}
$$

which come from the general equations (8) and (9). Note that this Lagrangian depends on the function $f^{\prime}$ which appears in the Ansatz for the form, but not on $f$ itself. Therefore the equation of motion for $f$ can be integrated to give the first integral

$$
f^{\prime}=u E^{-1} e^{6 \alpha_{0}+\frac{1}{2} \phi}
$$

with an integration constant $u$. From the Lagrangian (44) we can compute the equations of motion for $\alpha_{0}, \alpha_{1}$ and $\phi$. Using eq. (46) to replace $f^{\prime}$ in these equations, we arrive at

$$
\begin{aligned}
\frac{d}{d \tau}\left(E\left(-12 \alpha_{0}^{\prime}-36 \alpha_{1}^{\prime}\right)\right)+3 u^{2} E^{-1} e^{6 \alpha_{0}-\phi / 2} & =0 \\
\frac{d}{d \tau}\left(E\left(-36 \alpha_{0}^{\prime}-60 \alpha_{1}^{\prime}\right)\right) & =0 \\
\frac{d}{d \tau}\left(E \phi^{\prime}\right)-\frac{1}{4} u^{2} E^{-1} e^{6 \alpha_{0}-\phi / 2} & =0 .
\end{aligned}
$$


Let us compare these equations with the general ones given in the moduli space formalism in (13), (14) and (17). We see that they can be indeed written in this form if we set

$$
G=\left(\begin{array}{ccc}
-12 & -36 & 0 \\
-36 & -60 & 0 \\
0 & 0 & 1
\end{array}\right)
$$

and

$$
U=\frac{1}{2} u^{2} e^{6 \alpha_{0}-\frac{1}{2} \phi} .
$$

The matrix $G$ above is consistent with the general formula (12) with $d_{0}=3, d_{1}=6$ and $D=10$. In eq. (17) we introduced a systematic way of writing the effective potential by introducing a characteristic vector $\mathbf{q}_{r}$ for each form. From eq. (49) we read off that this vector is given by $\mathbf{q}=(6,0,-1 / 2)$ for our example. This coincides with what one gets by applying the general rule (6) to the breakup $\left(d_{0}, d_{1}\right)=(3,6)$ and a $\delta=3$ form in the 3 -dimensional subspace. The dilaton coupling $a(\delta)$ for a RR 3-form needed in eq. (6) follows from eq. (3) to be $a(\delta)=-1 / 2$.

In section 2 we also defined a scalar product (21) on the space of vectors $\mathbf{q}$ using the inverse of G. From eq. (48) $G^{-1}$ is given by

$$
G^{-1}=\left(\begin{array}{ccc}
\frac{5}{48} & -\frac{1}{16} & 0 \\
-\frac{1}{16} & \frac{1}{48} & 0 \\
0 & 0 & 1
\end{array}\right) .
$$

which agrees with the general formula (22) for $\left(d_{0}, d_{1}\right)=(3,6)$ and $D=10$. One can easily verify that $\left\langle\mathbf{q}, \mathbf{q}>=\mathbf{q} G^{-1} \mathbf{q}=4\right.$. Therefore $\mathbf{q}$ is indeed a spacelike vector, in agreement with the general result (30) which showed that this is true for all vectors obtained from type II forms.

The main problem in solving the system of equations (47) is the existence of two different exponentials, one coming from $E$, eq. (45), the other coming from the effective potential $U$, eq. (49). Fortunately, we have a gauge freedom (time reparameterization invariance) encoded in $N$ which we can use to get rid of one of the exponentials. In the next section we will use this freedom to set $E=1$. Here, however, we choose the another possibility, namely to gauge away the potential by setting $E=\exp \left(6 \alpha_{0}-\phi / 2\right)$. Given the definition of $E$ in eq. (45), this implies

$$
N=\exp \left(-3 \alpha_{0}+6 \alpha_{2}+\phi / 2\right)
$$

in accordance with the general formula (24) for $\mathbf{d}=(3,6,0)$ and $\mathbf{q}=(6,0,-1 / 2)$. With this choice, the equations of motion (47) turn into

$$
\begin{aligned}
& \frac{d}{d \tau}\left(e^{6 \alpha_{1}-\phi / 2}\left(2 \alpha_{1}^{\prime}+6 \alpha_{2}^{\prime}\right)\right)=\frac{u^{2}}{2} \\
& \frac{d}{d \tau}\left(e^{6 \alpha_{1}-\phi / 2}\left(3 \alpha_{1}^{\prime}+5 \alpha_{2}^{\prime}\right)\right)=0
\end{aligned}
$$




$$
\begin{aligned}
\frac{d}{d \tau}\left(e^{6 \alpha_{1}-\phi / 2} \phi^{\prime}\right) & =\frac{u^{2}}{2} \\
e^{6 \alpha_{1}-\phi / 2}\left(6 \alpha_{1}^{\prime 2}+36 \alpha_{1}^{\prime} \alpha_{2}^{\prime}+30 \alpha_{2}^{\prime 2}-\phi^{\prime 2}\right) & =\frac{u^{2}}{2} .
\end{aligned}
$$

This is consistent with the general form (26) found for models with one term in the potential. Taking appropriate linear combinations of the first three equations we can derive an equation for the remaining exponent $6 \alpha_{0}-\phi / 2$, which can be solved. Then $\alpha_{0}, \alpha_{1}, \phi$ can be expressed in terms of this solution. In this way one arrives at a general solution of the form (26) with coefficients c given by

$$
\mathbf{c}=\left(\frac{5}{16},-\frac{3}{16},-\frac{1}{4}\right)
$$

and the following constraints on the integration constants

$$
\begin{aligned}
6 w_{1}-\frac{1}{2} w_{3} & =1 \\
12 w_{1}^{2}+72 w_{1} w_{2}+60 w_{2}^{2} & =w_{3}^{2} \\
6 k_{1}-\frac{1}{2} k_{3} & =\ln \left(u^{2}\right) .
\end{aligned}
$$

One may arrive at the same result by inserting $\mathbf{q}=(6,0,-1 / 2)$ and $G^{-1}$ from eq. (50) into the general formulae (27), (28). Recall that the time parameter $\tau$ is restricted by $0<\tau<\tau_{1}$.

To discuss the cosmology of these solutions we must perform a transformation to comoving time $t$. To do this, we need the explicit form of the gauge parameter $N$ which we find by inserting the solution (26) with (53), (54) into eq. (51)

$$
N=e^{-3 k_{0}+6 k_{1}+k_{2} / 2}\left|\tau_{1}-\tau\right|^{-x+\Delta-1}|\tau|^{x-1} .
$$

Here $x=3 w_{0}+6 w_{1}$ and $\Delta=-3 / 16$. The quantities $x, \Delta$ have been generally defined in eq. (35), (36) and their values can be easily reproduced by inserting $\mathbf{d}=(3,6,0)$ and $\mathbf{c}$ from eq. (53). The range of comoving time obtained by integrating $d t=N(\tau) d \tau$ over $0<\tau<\tau_{1}$ crucially depends on the singularities in $N$. Eq. (55) shows that there are potential singularities at $\tau=0$ and $\tau=\tau_{1}$. Their appearance is controlled by the value of $x$.

Let us therefore analyze which values of $x$ are allowed. The first two constraints (54) may be solved to express, for example, $w_{0}$ and $w_{1}$ as a function of $w_{2}$. This shows that $x$ depends on one free parameter only. Furthermore, since the second constraint (54) is quadratic in $w_{I}$, we find two branches satisfying $x<\Delta=-3 / 16$ and $x>0$, respectively. We refer to these two branches as the $(-)$ and the $(+)$ branch. From eq. (55) we see that $0<\tau<\tau_{1}$ is indeed mapped to the comoving time ranges given in eq. (29); that is to $\left[-\infty, t_{1}\right]$ for the $(-)$ branch and to $\left[t_{0}, \infty\right]$ for the $(+)$ branch $\left(t_{0}, t_{1}\right.$ are integration constants). Moreover, the scalar curvature (37) has a future timelike singularity in the $(-)$ branch and a past timelike singularity in the $(-)$ branch. Both types of solutions are therefore not extendible. 
Information about the evolution of the two subspaces and the dilaton can be obtained form the respective Hubble parameters $\mathbf{H}=\dot{\boldsymbol{\alpha}}$ written as a function of comoving time. They can be calculated if $\tau \simeq 0$ or $\tau \simeq \tau_{1}$ since $N$ in eq. (55) can be integrated in these limits. Doing this for our example by using eq. (26), (53), (54) and $N, \tau(t)$ calculated from eq. (55), we find $\mathbf{H}$ to be of the Kaluza-Klein form (39), (40). The expansion coefficients $\mathbf{p}$ depend on the integration constants $\mathbf{w}$ as in eq. (42).

In fact, using the first two constraints (54) we may rewrite $\mathbf{p}$ as a function of $w_{2}$ only, as we did for the parameter $x$ before. The asymptotic expansion properties of our example therefore depend on one free parameter only. Instead of giving the explicit formulae, which are not particularly enlightening, let us give a graphical representation of $\mathbf{p}=\mathbf{p}\left(w_{2}\right)$. We concentrate on the $(+)$ branch (the expansion coefficients in the $(-)$ branch can be worked out analogously) where $t_{0}<t<\infty$ and the asymptotic regions are characterized by $t \simeq t_{0}(\tau \simeq 0)$ and $t \rightarrow \infty\left(\tau \simeq \tau_{1}\right)$. The results are given in fig. $1\left(t \simeq t_{0}\right)$ and fig. $2(t \rightarrow \infty)$. In both figures $\left|p_{0}\right|,\left|p_{1}\right|<1$ always, which illustrates our general result that the expansion in the $(+)$ branch is always subluminal (cf. eq. (41)).

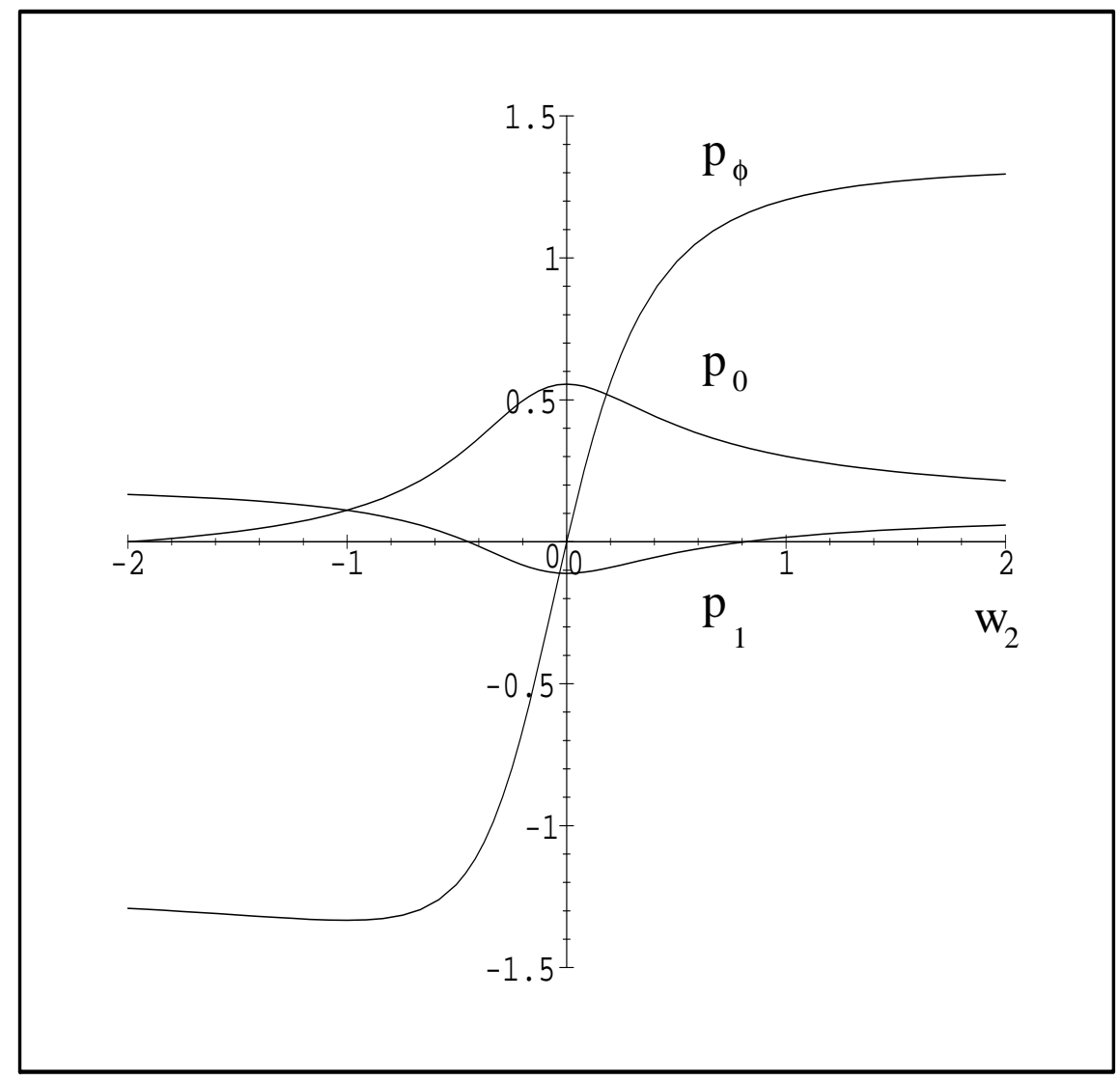

Fig 1: Expansion coefficients for the $(+)$ branch at $t \simeq t_{0}$.

We see that an early expansion of the 3 -dimensional subspace $\left(p_{0}>0\right.$ in fig. 1$)$ is turned 
into a contraction as $t \rightarrow \infty\left(p_{0}<0\right.$ in fig. 2) for a wide range in $w_{2}$. This can be understood from the $\alpha_{0}$ dependence of the effective potential (49). Moreover, the 6-dimensional space is always expanding as $t \rightarrow \infty\left(p_{1}>0\right.$ in fig. 2). In a more realistic model, such an expansion should be stopped by, for instance, a nonperturbative potential for the modulus $\alpha_{1}$.

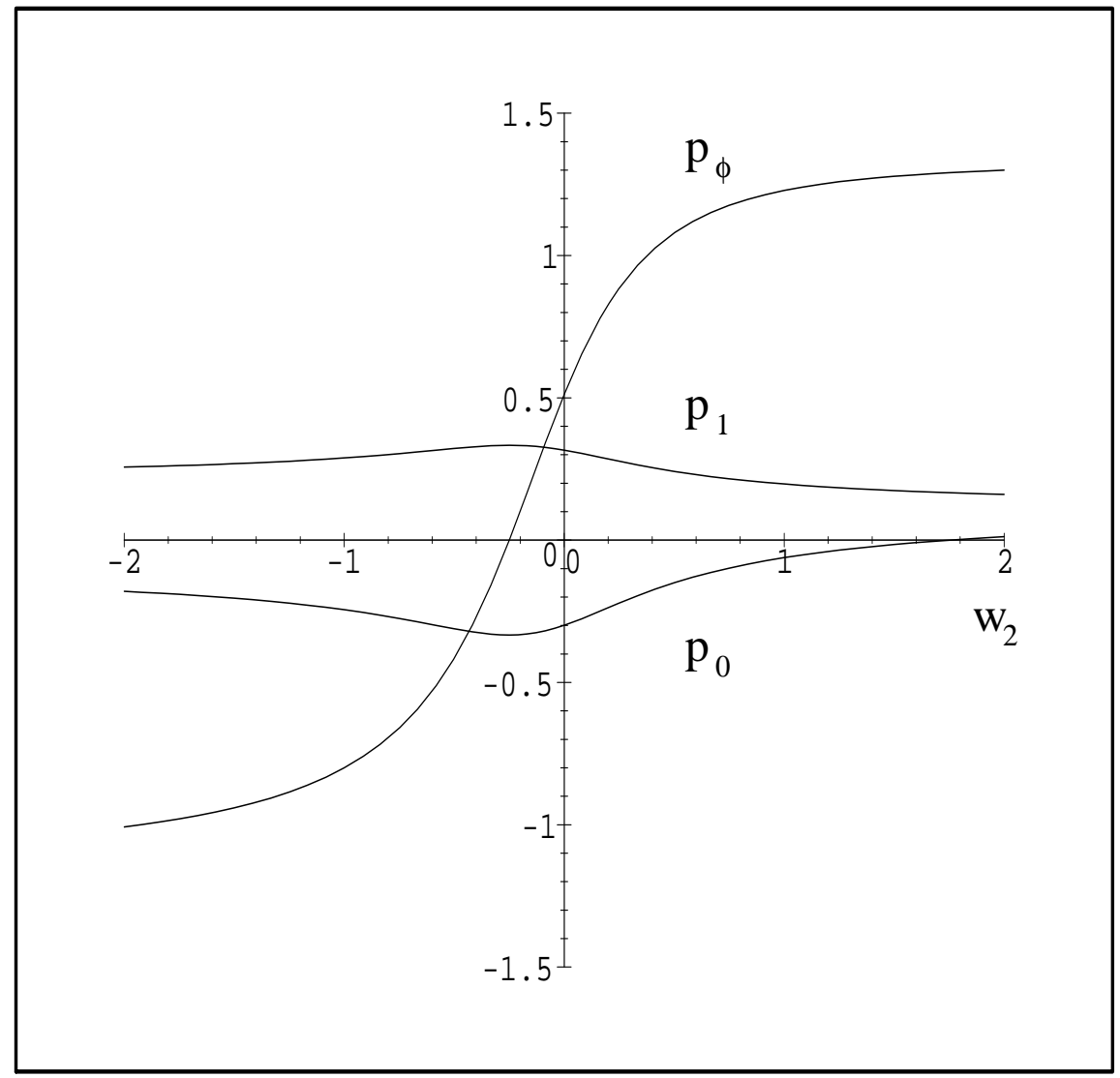

Fig 2: Expansion coefficients for the $(+)$ branch at $t \rightarrow \infty$.

\section{More general potentials - Toda models}

So far, we have explicitly solved the case with just one form turned on. As already mentioned, models with two or more forms are only soluble if they correspond to Toda models. In this section we will establish the relation to Toda models, and discuss two classes of solutions with some examples.

One recalls that the main result of section 2 was that the problem of finding cosmological solutions could be reduced to solving for the motion of a particle in a moduli space with a particular potential. The coordinates in the moduli space were an $n+1$ component vector $\boldsymbol{\alpha}=\left(\alpha_{i}, \phi\right)$ containing the scale factors $\alpha_{i}, i=0, \ldots, n-1$ of the $n$ maximally symmetric subspaces in which we divided the $D$-dimensional space and the dilaton. The dynamics of $\boldsymbol{\alpha}$ were then described by 
the particle equations of motion (13), (14) with the potential given by (17). The inverse metric on the moduli space (22) defined a natural scalar product $<,>$ (21) for covariant vectors living in the moduli space (such as $\mathbf{q}$ ). We also recall that the metric had Minkowskian signature. To make contact with Toda models we would like to write all the moduli-space equations of motion in terms of the scalar product. To this end we introduce a "lowered-index" covariant vector corresponding to the contravariant coordinate vector $\boldsymbol{\alpha}$ by

$$
\boldsymbol{\beta}=G \boldsymbol{\alpha}
$$

Lets us also choose to work in the gauge

$$
N=\exp (<\mathbf{d}, \boldsymbol{\beta}>)
$$

which implies that $E=1$. In this gauge, the moduli space Lagrangian (16) written in terms of $\boldsymbol{\beta}$ reduces to a form $\mathcal{L}_{0}$. The constraint (14) can then be expressed as the vanishing of the corresponding Hamiltonian $\mathcal{H}_{0}$ so that the whole system is described by

$$
\begin{aligned}
\mathcal{L}_{0} & =\frac{1}{2}<\boldsymbol{\beta}^{\prime}, \boldsymbol{\beta}^{\prime}>-U \\
\mathcal{H}_{0} & =\frac{1}{2}<\boldsymbol{\beta}^{\prime}, \boldsymbol{\beta}^{\prime}>+U=0 \\
U & =\frac{1}{2} \sum_{r=1}^{m} u_{r}^{2} \exp \left(<\mathbf{q}_{r}, \boldsymbol{\beta}>\right) .
\end{aligned}
$$

The prime denotes the derivative with respect to the time parameter $\tau$. Recall that each of the $m$ terms in the potential $U$ corresponds to a certain Ansatz for a form specified by the respective vector $\mathbf{q}_{r}, r=1, \ldots, m$.

A general solution for this system can be found if it corresponds to a Toda model [31]; that is, if the matrix

$$
A_{r s}=\frac{<\mathbf{q}_{r}, \mathbf{q}_{s}>}{q}
$$

can be identified with the Cartan matrix of a semi-simple Lie group $G$ for some constant $q$ ․ Note that $A$ is an $m \times m$ matrix, where $m$ is the number of forms excited, each specified by a different $\mathbf{q}_{r}$.

Let us briefly show that this property does not depend on the particular coordinate system in moduli space we have chosen. Under a linear coordinate transformation $\boldsymbol{\alpha} \rightarrow \overline{\boldsymbol{\alpha}}=P \boldsymbol{\alpha}$, the covariant vectors $\boldsymbol{\beta}$ and $\mathbf{q}_{r}$ transform as $\boldsymbol{\beta} \rightarrow \overline{\boldsymbol{\beta}}=P^{-1^{T}} \boldsymbol{\beta}$ and $\mathbf{q}_{r} \rightarrow \overline{\mathbf{q}}_{r}=P^{-1^{T}} \mathbf{q}_{r}$, while the new inverse metric is given by $\bar{G}^{-1}=P G^{-1} P^{T}$. Clearly all scalar products are unchanged. Thus, the matrix $A_{r s}$ is invariant under such an operation and the property of being (or not being) a Toda model is coordinate independent. In particular, the transition from the Einstein to the string frame

\footnotetext{
${ }^{3}$ If the group is semisimple $q$ can be different for each simple factor.
} 
discussed in the beginning of section 2 can be described by such a change of coordinates in moduli space. This shows that our decision to work in the Einstein frame does not affect the essentials of the present discussion. A similar remark applies to duality transformations within the class of models considered here, which we also expect to act as linear transformations on $\boldsymbol{\beta}$.

Are there really such Toda models among the examples one obtains from type II theories? To answer this question systematically, and to give a simple criterion to decide in each specific case, we will now compute the possible entries of the matrix $A$. We consider two forms of degree $\delta_{1}$ and $\delta_{2}$ with vectors $\mathbf{q}_{1}, \mathbf{q}_{2}$ as defined in eq. (6) and (7). From eq. (21), their scalar product is given by

$$
\begin{aligned}
<\mathbf{q}_{1}^{(\mathrm{el}, \mathrm{NS})}, \mathbf{q}_{2}^{(\mathrm{el}, \mathrm{NS})}> & =2 \delta_{12} \\
<\mathbf{q}_{1}^{(\mathrm{el}, \mathrm{R})}, \mathbf{q}_{2}^{(\mathrm{el}, \mathrm{R})}> & =2 \delta_{12}-\delta_{1}-\delta_{2}+\frac{D-2}{2} \\
<\mathbf{q}_{1}^{(\mathrm{el}, \mathrm{R})}, \mathbf{q}_{2}^{(\mathrm{el}, \mathrm{NS})}> & =2 \delta_{12}-2
\end{aligned}
$$

and

$$
\begin{aligned}
& <\mathbf{q}_{1}^{(\mathrm{sol})}, \mathbf{q}_{2}^{(\mathrm{sol})}>=<\mathbf{q}_{1}^{(\mathrm{el})}, \mathbf{q}_{2}^{(\mathrm{el})}>-2 \\
& <\mathbf{q}_{1}^{(\mathrm{el})}, \mathbf{q}_{2}^{(\mathrm{sol})}>=-<\mathbf{q}_{1}^{(\mathrm{el})}, \mathbf{q}_{2}^{(\mathrm{el})}>
\end{aligned}
$$

where $\delta_{12}=\sum_{i=0}^{n-1} \epsilon_{i}^{(1)} \epsilon_{i}^{(2)} d_{i}$ is the "spatial overlap" of the two forms, that is the dimension of the subspace in which both forms are non-zero. As before, the cosmological constant of massive IIA is analogous to a $\mathrm{RR}(D-1)$-form. The above expressions show that the entries of the matrix $A$ are small integer numbers (at least for $D=10$ ) of either sign which are just the right properties to construct a Cartan matrix. For a number of models $A$ can indeed be identified with a Cartan matrix. Explicit examples of this have been discussed in ref. [8]. Here we are mainly interested in the general structure of the solutions.

\subsection{Orthogonal vectors $q$}

A simple class of soluble models [32] is characterized by orthogonal vectors $\mathbf{q}_{r}$; that is, $<\mathbf{q}_{r}, \mathbf{q}_{s}>=0$ for $r \neq s$. Then the matrix $A$ is diagonal and can, via appropriate rescalings, be identified with the Cartan matrix of $S U(2)^{m}$.

Before we solve the equations of motion, let us show that such a situation can indeed occur within type II theories. We consider two forms of type IIA, namely an elementary 3-form specified by $\mathbf{q}_{1}$ and a solitonic 1 -form specified by $\mathbf{q}_{2}$ occupying different subspaces. In the language of eq. (61) that means $\delta_{1}=3, \delta_{2}=1$ and $\delta_{12}=0$ (no overlap). Then from eq. (63) and (61) we have $<\mathbf{q}_{1}, \mathbf{q}_{1}>=<\mathbf{q}_{2}, \mathbf{q}_{2}>=4$ and $<\mathbf{q}_{1}, \mathbf{q}_{2}>=0$ and, hence, we have orthogonal vectors $\mathbf{q}_{r}$.

Let us now solve the equations of motion. It is useful to introduce an orthonormal basis $\mathbf{e}_{i}$, 
$i=0, \ldots, n$ in moduli space satisfying

$$
<\mathbf{e}_{i}, \mathbf{e}_{j}>=\eta_{i} \delta_{i j}
$$

with $\eta_{0}=-1$ and $\eta_{i}=1$ for $i>0$. In the following, we will use indices $i, j, \ldots=0, \ldots, n$ to label these basis vectors. Remember that the scalar product has a Minkowskian signature so that one of these vectors, $\mathbf{e}_{0}$, is normalized to -1 . We have assumed that the vectors $\mathbf{q}_{r}, r=1, \ldots, m$ are orthogonal. This allows us to identify some of the basis vectors $\mathbf{e}_{i}$, with a normalized version of the $\mathbf{q}_{r}$

$$
\mathbf{e}_{r}=\frac{-\mathbf{q}_{r}}{q_{r}}, \quad q_{r}=\sqrt{\left|<\mathbf{q}_{r}, \mathbf{q}_{r}>\right|}, \quad r=1, \ldots, m .
$$

Note here, that the $\mathbf{q}_{r}$ are orthogonal spacelike vectors. This implies that their number (the number of forms) is less than the dimension of the moduli space; that is, $m \leq n$. Furthermore, none of the $\mathbf{q}_{r}$ can be identified with the timelike basis vector $\mathbf{e}_{0}$. The basis vectors (64) therefore fall into two groups, those which can be identified with forms and those which cannot. Correspondingly, we partition the index $i=0, \ldots, n$ as $i=(a, r)$, where we use the same index $r=1, \ldots, m$ as for the forms to label the basis vectors proportional to $\mathbf{q}_{r}$ and the index $a=0, m+1, \ldots, n$ to label the other basis vectors.

We expand the "dynamical" vector $\boldsymbol{\beta}$ in terms of the basis

$$
\boldsymbol{\beta}=\sum_{i=0}^{n} \rho_{i} \mathbf{e}_{i}
$$

with (time dependent) expansion coefficients $\rho_{i}(\tau)$. Inserting this expansion into $\mathcal{L}_{0}$ given in eq. (58), we find a complete decoupling in the eigenmodes $\rho_{i}$ and the resulting equations of motion

$$
\begin{aligned}
\rho_{r}^{\prime \prime} & =\frac{1}{2} q_{r} u_{r}^{2} e^{-q_{r} \rho_{r}} \\
\rho_{a}^{\prime \prime} & =0 .
\end{aligned}
$$

Their solution given by

$$
\begin{aligned}
\rho_{r} & =q_{r}^{-1} \ln \left(g_{r}\right) \\
\rho_{a} & =k_{a}\left(\tau-\tau_{a}\right)
\end{aligned}
$$

with where we define

$$
g_{r}=K_{r} \cosh ^{2}\left(z_{r}\right), \quad K_{r}=\frac{u_{r}^{2}}{k_{r}^{2}}, \quad z_{r}=\frac{1}{2}\left|k_{r}\right| q_{r}\left(\tau-\tau_{r}\right),
$$

Here $\tau$ runs over the full real axis and $k_{i}$ and $\tau_{i}$ are integration constants. Bringing everything together, the solution for the original vector $\boldsymbol{\alpha}$ reads

$$
\boldsymbol{\alpha}=\sum_{i=0}^{n} \rho_{i} G^{-1} \mathbf{e}_{i}
$$

with $\rho_{i}$ as indicated above. 
The application to an explicit example is straightforward. First one should calculate the matrix $G^{-1}$ from eq. (22). Then one determines the vectors $\mathbf{q}_{r}$ specifying the forms from eq. (6), (7) and calculates the basis vectors $\mathbf{e}_{r}, r=1, \ldots, m$ via eq. (65). Finally, one extends this subset of basis vectors to a full orthogonal basis of the moduli space satisfying eq. (64). All these quantities inserted into eq. (71) provide the explicit solution.

So far we have not considered the Hamiltonian constraint $\mathcal{H}_{0}=0$ in eq. (58). For the above solution it leads to

$$
\mathcal{H}_{0}=\frac{1}{2} \sum_{i=0}^{n} \eta_{i} k_{i}^{2}=0 .
$$

Correspondingly, the constants $k_{i}$ can be interpreted as the "energy" contribution of the modes $\rho_{i}$. To understand the meaning of the "time shifts" $\tau_{r}$ it is instructive to compute the potential $U$ for the above solution

$$
U=\frac{1}{2} \sum_{r=1}^{m} u_{r}^{2} g_{r}^{-1}=\frac{1}{2} \sum_{r=1}^{m} k_{r}^{2}\left[\cosh \left(z_{r}\right)\right]^{-2} .
$$

It indicates that the form $r$ is operative around $\tau \simeq \tau_{r}$ and switched off otherwise so that we expect asymptotic Kaluza-Klein behavior at $\tau \rightarrow \pm \infty$. Suppose we time order the time shifts $\tau_{r}$; that is, $\tau_{r_{1}} \leq \tau_{r_{2}} \leq \ldots \leq \tau_{r_{m}}$. Then the system starting out at $\tau \rightarrow-\infty$ will go through a sequence of "transformations" each induced by one of the forms before it reaches the asymptotic region at $\tau \rightarrow \infty$. If the time differences $\tau_{r_{i+1}}-\tau_{r_{i}}$ are all of order one, then the forms will act almost simultaneously. However, if there is a long interval between two of the time shifts, for example $\tau_{r_{2}}-\tau_{r_{1}} \gg 1$, the potential is effectively turned off during much of the intermediate period $\tau_{r_{1}}<\tau<t_{r_{2}}$. Depending on the number of long intervals, we may have up to $m-1$ of these intermediate Kaluza-Klein regions.

Though our solutions cannot be generally expressed in terms of the comoving time in closed form, this can be done in the Kaluza-Klein regions. For the gauge parameter $N$ we find from eq. (57) and (56)

$$
N=\exp \left(\sum_{i} \Delta_{i} \rho_{i}\right), \quad \Delta_{i}=<\mathbf{d}, \mathbf{e}_{i}>.
$$

Using the explicit expression for $\rho_{i}$, eq. (69), this can be approximated in any one of the KaluzaKlein regions as

$$
N \simeq N_{0} e^{\nu \tau}, \quad \nu=\sum_{a} k_{a} \Delta_{a}+\sum_{r}( \pm)\left|k_{r}\right| \Delta_{r}
$$

Note that there is a sign freedom in the second term of the second equation. This freedom serves to specify the asymptotic region for which $N$ is computed. Let us concentrate on the asymptotic region specified by $\tau_{r_{i}}<\tau<\tau_{r_{i+1}}$. Then all terms with $r=r_{1}, \ldots, r_{i}$ get a + sign and all terms with $r=r_{i+1}, \ldots, r_{m}$ get a - sign. More intuitively, a term $r$ gets a + sign if the $r$ th form has been "passed" and a - sign otherwise. Of particular importance are the two parameters $\nu_{ \pm}$ corresponding to $\tau \rightarrow \pm \infty$ which determine the relevant range of the comoving time $t$. We get $\nu_{+}$ 
from the above formula for $\nu$ by taking all signs to be + and $\nu_{-}$by taking all signs to be - . From eq. (75), the quantities $\nu_{ \pm}$do not seem to be restricted in range. We have, however, to consider the Hamiltonian constraint (72) on the integration constants $k_{i}$. Using this constraint, we find for all explicit examples that $\nu_{+}$and $\nu_{-}$always have the same sign. Then integration of $d t=N(\tau) d \tau$ with $N$ as in eq. (75) leads to two unconnected branches with

$$
\tau \rightarrow t \in\left\{\begin{array}{lll}
{\left[-\infty, t_{1}\right],} & \nu_{ \pm}<0 & (-) \text { branch } \\
{\left[t_{0},+\infty\right],} & \nu_{ \pm}>0 & (+) \text { branch }
\end{array}\right.
$$

The Hubble parameters in each Kaluza-Klein region take the standard form (39), with the expansion coefficients $\mathbf{p}$ expressed in terms of the integration constants as

$$
\mathbf{p}=\frac{1}{\nu}\left[\sum_{a} k_{a} G^{-1} \mathbf{e}_{a}+\sum_{r}( \pm)\left|k_{r}\right| G^{-1} \mathbf{e}_{r}\right] .
$$

The signs in the second term have to be chosen according to the same rule as above. Depending on the choice of these signs, eq. (77) specifies the expansion parameters in each of the up to $m+1$ asymptotic regions. To get this result we have used the definition of $\mathbf{H}$ in eq. (39), the solution (71) and $N, t(\tau)$ to be calculated from eq. (75). Clearly, these coefficients fulfill the two equations (40) characteristic for a Kaluza-Klein region. Therefore, the discussion of cosmological properties given in section 3 based on these equations applies here as well. In particular, the expansion in the $(-)$ branch is superinflating for $p_{i}<0$ and the expansion in the $(+)$ branch is always subluminal since $\left|p_{i}\right|<1$.

In summary, we have found the direct generalization of the simple case with one form : the solutions split into two different branches $(+),(-)$ with properties similar to those discussed in section 3. We also have two Kaluza-Klein regions, one at early and one at late time, in each respective branch. If all time shifts $\tau_{r}$ are of the same order, these two regions are the only KaluzaKlein regions, as in the case of one form. The transition, however, is then generated by the combined effect of all forms. If there are long intervals between the $\tau_{r}$ we may have up to $m-1$ intermediate Kaluza-Klein regions, where $m$ is the number of forms. In each region the forms are effectively turned off. A transition is generated when one or more of the forms become operative.

\subsection{A Toda example}

Let us illustrate the general results above by an explicit example. We break up the space as $\mathbf{d}=(3,2,4,0)$ and consider an elementary IIA 3 form in the $d_{0}=3$ subspace and a solitonic IIA 1 form in the $d_{1}=2$ subspace. From the eqs. (6) and (7) we find the corresponding vectors $\mathbf{q}_{1}=(6,0,0,-1 / 2)$ for the 3 form and $\mathbf{q}_{2}=(6,0,8,3 / 2)$ for the 1 form. In the notation of eq. (61) we therefore have $\delta_{1}=3, \delta_{2}=1$ and $\delta_{12}=0$. Inserting these quantities into the eqs. (61), (62) and 
(63) we find $<\mathbf{q}_{1}, \mathbf{q}_{1}>=<\mathbf{q}_{2}, \mathbf{q}_{2}>=4$ and $<\mathbf{q}_{1}, \mathbf{q}_{2}>=0$. This shows that our example indeed corresponds to an orthogonal model. By using the inverse matrix

$$
G^{-1}=\left(\begin{array}{cccc}
\frac{5}{48} & -\frac{1}{16} & -\frac{1}{16} & 0 \\
-\frac{3}{16} & \frac{3}{16} & -\frac{1}{16} & 0 \\
-\frac{1}{16} & -\frac{1}{16} & \frac{1}{16} & 0 \\
0 & 0 & 0 & 1
\end{array}\right)
$$

obtained from eq. (22) this can also be verified explicitly. Apart from $G^{-1}$ we need the set $\mathbf{e}_{i}$, $i=0,1,2,3$ of orthogonal vectors in moduli space to get the explicit solution from eq. (71). The two basis vectors $\mathbf{e}_{1}, \mathbf{e}_{2}$ in the direction of the forms follow easily from eq. (65) by normalizing $\mathbf{q}_{1}$ and $\mathbf{q}_{2}$. The two remaining vectors $\mathbf{e}_{0}, \mathbf{e}_{3}$ should be chosen to complete this to a basis of the moduli space. A possible choice for the complete basis is

$$
\begin{aligned}
& \mathbf{e}_{0}=(6,2,8,0) \\
& \mathbf{e}_{1}=\left(-3,0,0, \frac{1}{4}\right) \\
& \mathbf{e}_{2}=\left(-3,0,-4,-\frac{3}{4}\right) \\
& \mathbf{e}_{3}=\sqrt{6}\left(1,0,2,-\frac{1}{4}\right) .
\end{aligned}
$$

It is straightforward to verify that this set of vectors indeed fulfills the normalization condition (64). Inserting $G^{-1}$ and $\mathbf{e}_{i}$ into eq. (71), along with the functions $\rho_{i}$, gives the explicit solution for our example. It follows from eq. (69) that the functions $\rho_{i}, i=0,1,2,3$ read

$$
\rho_{r}=\frac{\ln \left(g_{r}\right)}{q_{r}}
$$

for $r=1,2$ where $g_{r}$ is given in eq. (70), and

$$
\rho_{a}=k_{a}\left(\tau-\tau_{a}\right)
$$

for $a=0,3$. The effective potential $U$, eq. (73), specializes to

$$
U=\frac{1}{2}\left(k_{1}^{2} \cosh ^{-2}\left(z_{1}\right)+k_{2}^{2} \cosh ^{-2}\left(z_{2}\right)\right)
$$

for our example, where $z_{r}$ has been defined in eq. (70). Note that the first term in this potential is related to the 3 form and the second one to the 1 form.

For a discussion of cosmological properties the numbers $\Delta_{i}$ defined in eq. (74) are needed (see for example eq. (75)). From the above basis vectors they are easily computed to be $\Delta_{0}=-1$, $\Delta_{1}=3 / 16, \Delta_{2}=7 / 16$ and $\Delta_{3}=-3 \sqrt{6} / 16$. Now we have explicitly given all quantities required to compute the gauge parameter $N$, eq. (75), and the expansion parameters $\mathbf{p}$, eq. (77), as a function of the integration constants $k_{i}$. Note that the Hamiltonian constraint (72) should be used to eliminate one of these integration constants. 
Let us discuss the various cases that can occur within our model. Recall first that each form $r=1,2$ is operative at $\tau \simeq \tau_{r}$ only, where $\tau_{r}$ are free integration constants. The dashed line in fig. 3 shows the potential (82) for $\tau_{1}=-1, \tau_{2}=1$, so that the difference in the time shifts is of order one. It can be seen that the effect of the two forms cannot be separated in time in this case. We have only two asymptotic regions, $\tau \rightarrow-\infty$ and $\tau \rightarrow \infty$. As an example, we want to compute the quantities $\nu_{ \pm}$, which specify the behaviour of the gauge parameter $N$, in these regions. Using the constants $\Delta_{i}$ given above we find from eq. (75)

$$
\nu_{-}=-k_{0}-\frac{3 \sqrt{6}}{16} k_{3}-\frac{3}{16}\left|k_{1}\right|-\frac{7}{16}\left|k_{2}\right|
$$

for the asymptotic region $\tau \rightarrow-\infty$. Correspondingly, for the region $\tau \rightarrow \infty$ we find

$$
\nu_{+}=-k_{0}-\frac{3 \sqrt{6}}{16} k_{3}+\frac{3}{16}\left|k_{1}\right|+\frac{7}{16}\left|k_{2}\right|
$$

Note that the $r=1,2$ terms are both negative for $n_{-}$and both positive for $n_{+}$. This is in agreement with the general description of the sign choice, which we have given below eq. (75). The Hamiltonian constraint (72) reads

$$
\mathcal{H}_{0}=-k_{0}^{2}+k_{1}^{2}+k_{2}^{2}+k_{3}^{2}=0
$$

and can be used to eliminate, for example, $k_{0}$ from the above expressions for $\nu_{ \pm}$. Doing this, it can be seen explicitly, that $\nu_{+}$and $\nu_{-}$always have the same sign, independent on the values of $k_{1}, k_{2}, k_{3}$. As stated before, this leads to the two branches indicated in eq. (76). The computation of the expansion coefficients $\mathbf{p}$ in eq. (77) can be carried out in a similar way. We will, however, not give the explicit formulae here.

If $\left|\tau_{2}-\tau_{1}\right| \gg 1$ another intermediate Kaluza-Klein region appears. This is illustrated by the solid line in fig. 3 where we have plotted the potential $U$, eq. (73), for $\tau_{1}=-10$ and $\tau_{2}=10$. For this choice of $\tau_{r}$ the system in the intermediate region has already been influenced by the 3 form but not yet by the 1 form (of course one can have the converse situation by taking $\tau_{1} \gg \tau_{2}$ ). Correspondingly, for the expansion coefficients in eq. (77) one should take the + sign for the $r=1$ term and the - sign for the $r=2$ term. The expression for $\nu$ in this intermediate region therefore reads

$$
\nu=-k_{0}-\frac{3 \sqrt{6}}{16} k_{3}+\frac{3}{16}\left|k_{1}\right|-\frac{7}{16}\left|k_{2}\right|
$$

So far, we have carried out the discussion in terms of the time parameter $\tau$. Upon mapping to the comoving time $t$ via eq. ( $\sqrt{6})$, we have two different branches, the $(+)$ branch and the $(-)$ branch. The above discussion therefore applies to both branches. In particular, in both branches we may have either two or three Kaluza-Klein regions, depending on the values of the time shifts $\tau_{1}$ and $\tau_{2}$. Though we have not computed the expansion coefficients $\mathbf{p}$ explicitly, we know from their general properties (40) that the evolution in each of these Kaluza-Klein regions will be similar to 
what we have described in section 3 for one form only.

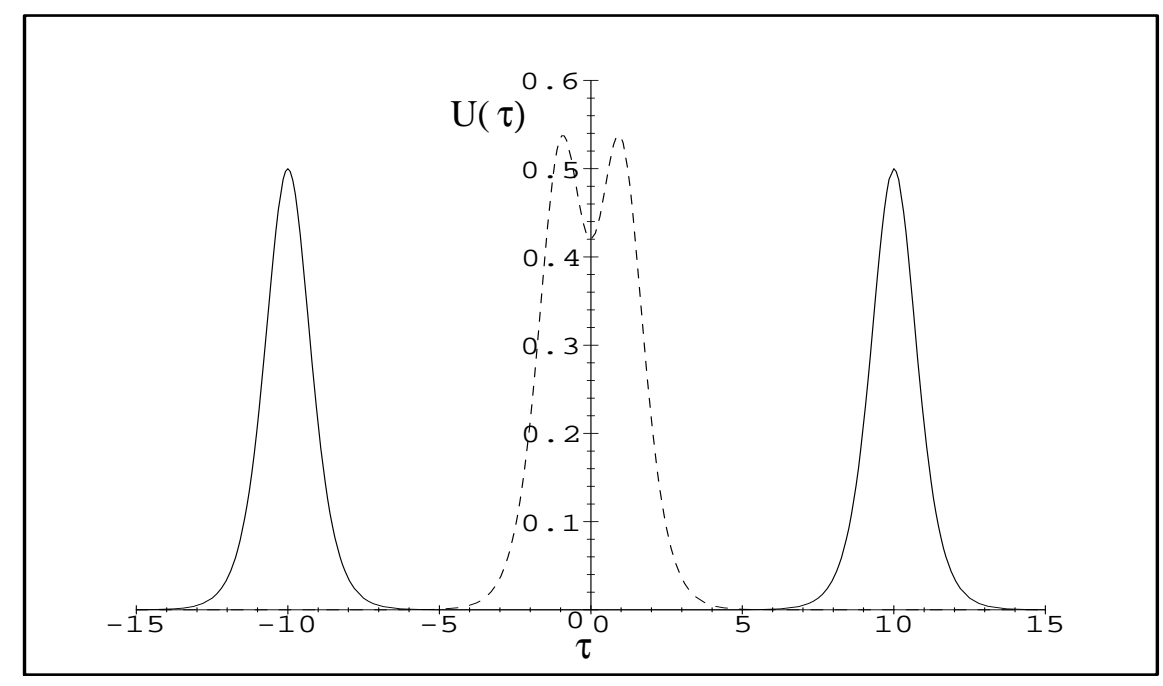

Fig 3: Effective potential $U$ as a function of the time parameter $\tau$ for the example described in the text. The solid line corresponds to $\tau_{1}=-10, \tau_{2}=10$, the dashed line to $\tau_{1}=-1, \tau_{2}=1$. For simplicity we have taken $k_{1}=k_{2}=1$.

\subsection{Nonorthogonal vectors $q$}

We now discuss the general case where the $\mathbf{q}_{i}$ vectors are not orthogonal. As in the previous subsection we start with an expansion (66) for $\boldsymbol{\beta}$ in terms of a basis $\mathbf{e}_{i}, i=0, \ldots, n$ in moduli space. Again, we would like to identify some of the basis vectors with the vectors $\mathbf{q}_{r}$ describing the forms. Therefore we define a basis with the following properties

$$
\begin{aligned}
<\mathbf{e}_{a}, \mathbf{e}_{b}> & =\eta_{a} \delta_{a b} \\
<\mathbf{e}_{a}, \mathbf{e}_{r}> & =0 \\
\mathbf{e}_{r} & =\frac{\mathbf{q}_{r}}{q} .
\end{aligned}
$$

Note that the basis vectors $\mathbf{e}_{r}$ are no longer orthogonal to each other. We remind the reader that the different types of indices were defined as $i, j, \ldots=0, \ldots, n$ for the whole set of basis vectors, $r, s, \ldots=1, \ldots, m$ for the basis vectors corresponding to forms and $a, b, \ldots=0, m+1, \ldots, n$ for the other basis vectors. With the above properties of $\mathbf{e}_{i}$, insertion of the expansion (66) into the Lagrangian (58) leads to

$$
\begin{aligned}
\mathcal{L}_{0} & =\frac{1}{2} \sum_{a} \eta_{\rho_{a}^{\prime}}^{2}+\frac{1}{2 q} \sum_{r, s} A_{r s} \rho_{r}^{\prime} \rho_{s}^{\prime}-U \\
U & =\frac{1}{2} \sum_{r} u_{r}^{2} \exp \left(\sum_{s} A_{r s} \rho_{s}\right) .
\end{aligned}
$$


Recall, that $A_{r s}$ defined in eq. (60) is assumed to be the Cartan matrix of a semi-simple Lie group $G$. From the above Lagrangian, we see that by introducing the basis $\mathbf{e}_{i}$ we have achieved a separation between the trivial modes which do not occur in the potential $U$ and the nontrivial ones in the directions of the vectors $\mathbf{q}_{r}$.

The solution of the equations of motion to be derived from $\mathcal{L}_{0}$ can be written in the form

$$
\begin{aligned}
\rho_{a} & =k_{a}\left(\tau-\tau_{a}\right) \\
\rho_{r} & =-2 A_{r r}{ }^{-1} \ln \left(g_{r}\right)
\end{aligned}
$$

with $g_{r}$ given by

$$
g_{r}=\sum_{\boldsymbol{\lambda} \in \Lambda_{r}} b_{r}(\boldsymbol{\lambda}) \exp (\boldsymbol{\lambda} \cdot \mathbf{k} \tau-\boldsymbol{\lambda} \cdot \boldsymbol{\tau}) .
$$

Here $\Lambda_{r}$ are the weights of the fundamental representations of the group $G, \mathbf{k}=\left(k_{r}\right)$ is a constant vector restricted to the Weyl chamber of $G$ and the set of time shifts $\boldsymbol{\tau}=\left(\tau_{r}\right)$ is arbitrary. As before the constants $k_{r}$ are subject to the Hamiltonian constraint. The coefficients $b_{r}(\boldsymbol{\lambda})$ are determined in terms of $k_{r}$ and properties of the group $G$ but the general expression [31] is not very helpful. In practice, one may just insert the above solution into

$$
\rho_{r}^{\prime \prime}+\frac{q}{2} u_{r}^{2} \exp \left(\sum_{s} A_{r s} \rho_{s}\right)=0
$$

to find $b_{r}(\boldsymbol{\lambda})$.

Let us proceed with the simplest nontrivial example $G=S U(3)$, that is, with a model having two forms excited and where the matrix $A$ takes the form 33]

$$
A=\left(\begin{array}{cc}
2 & -1 \\
-1 & 2
\end{array}\right) \text {. }
$$

How can such a model be realized within $D=10$ type II theories? We choose an elementary IIA 3-form with vector $\mathbf{q}_{1}$ and an elementary IIA NS 2-form with vector $\mathbf{q}_{2}$ such that they do not overlap in the spatial directions. In the language of eq. (61) we then have $\delta_{1}=3, \delta_{2}=2$ and $\delta_{12}=0$ which results in $\left.\left\langle\mathbf{q}_{1}, \mathbf{q}_{1}\right\rangle=<\mathbf{q}_{2}, \mathbf{q}_{2}\right\rangle=4$ and $\left\langle\mathbf{q}_{1}, \mathbf{q}_{2}\right\rangle=-2$. The relation (60) is therefore indeed fulfilled for the above $S U(3)$ Cartan matrix if we choose $q=2$.

The weight systems of the two fundamental $S U(3)$ representations are $\Lambda_{1}=\{(1,0),(-1,1),(0,-1)\}$ for $\mathbf{3}$ and $\Lambda_{2}=\{(0,1),(1,-1),(-1,0)\}$ for $\overline{\mathbf{3}}$. The constants $\mathbf{k}=\left(k_{1}, k_{2}\right)$ are constrained by $2 k_{1}-k_{2}>0,2 k_{2}-k_{1}>0$ ( $\mathbf{k}$ is in the Weyl chamber) which ensures that the argument $g_{r}$ of the logarithm in eq. (90) is positive. From eq. (92) we get for the coefficients $b_{r}(\boldsymbol{\lambda})$

$$
\begin{aligned}
& b_{1}(1,0)=q u_{1}^{2} \frac{2 k_{2}-k_{1}}{P} \quad b_{2}(0,1)=q u_{1}^{2} \frac{2 k_{1}-k_{2}}{P} \\
& b_{1}(-1,1)=q u_{1}^{2} \frac{k_{1}-k_{2}}{P} \quad b_{2}(1,-1)=q u_{2}^{2} \frac{k_{1}-k_{2}}{P} \\
& b_{1}(0,-1)=q u_{2}^{2} \frac{2 k_{1}-k_{2}}{P} \quad b_{2}(-1,0)=q u_{2}^{2} \frac{2 k_{2}-k_{1}}{P}
\end{aligned}
$$


with $P=\left(2 k_{2}-k_{1}\right)\left(k_{1}-k_{2}\right)\left(2 k_{1}-k_{2}\right)$. The Hamiltonian constraint $\mathcal{H}_{0}=0$ in eq. (58) turns into

$$
\mathcal{H}_{0}=\frac{1}{2} \sum_{a} \eta_{a} k_{a}^{2}+\frac{1}{2}\left(k_{1}^{2}-k_{1} k_{2}+k_{2}^{2}\right)=0 .
$$

by inserting the complete solution.

Though significantly more complicated, the structure of these solutions is similar to what we found in the case of orthogonal vectors q. For $\tau \rightarrow \pm \infty$ we have two asymptotic Kaluza-Klein regions. Having chosen appropriate constants $\mathbf{k}$ and $\boldsymbol{\tau}$ one may also have intermediate KaluzaKlein regions. The transition between these regions, however, is more complicated and the effect of the two forms can no longer be separated in time.

\section{$5 \quad$ Including curvature}

Thus far, we have assumed that the maximally symmetric subspaces into which we have split the total space are flat. In this section, we would like to show that the more general case of curved subspaces can easily be incorporated into our general framework. This is of particular interest because it allows us to investigate a class of cosmologies, directly related to black $p$-brane solutions, some examples of which have recently been given by Behrndt and Förste [16] and Poppe and Schwager [20].

Suppose, that instead of (5) we start with a metric

$$
d s^{2}=-N^{2}(\tau) d \tau^{2}+\sum_{i=0}^{n-1} e^{2 \alpha_{i}} d \Omega_{K_{i}}^{2},
$$

where $d \Omega_{K_{i}}^{2}$ is the metric of a $d_{i}$ dimensional space with constant curvature $K_{i}=-1,0$ or +1 . The effect of these terms is to create an additional potential $V_{c}$ for the scale factors $\alpha_{i}$. The effective Lagrangian (8) is modified to

$$
\mathcal{L}_{c}=\mathcal{L}-N^{2} E V_{c},
$$

where $V_{c}$ is explicitly given by

$$
V_{c}=-\sum_{i} 2 K_{i} e^{-2 \alpha_{i}}
$$

This potential is formally very similar to the one provided by the forms (see the eqs. (9)). It leads to terms of the form $u^{2} \exp (\mathbf{q} \cdot \boldsymbol{\alpha})$ in the effective potential $U$ which, in fact, we have already considered by using the general form (17) of $U$. All we have to do is to specify the vector $\mathbf{q}_{k}$ characterizing a curvature in the $k$ th subspace. From eq. (98) it follows that

$$
\mathbf{q}_{k}=\left(2 d_{0}, \ldots, 2\left(d_{k}-1\right), \ldots, 2 d_{n-1}, 0\right) .
$$

Moreover, the constant $u_{k}^{2}$ in front of such a curvature term in $U$ is given by $u_{k}^{2}=-2 K_{k}$ so that it can be of either sign depending on the sign of the curvature $K_{k}$. This is in contrast to terms in $U$ describing forms which are always positive. 
All methods presented in this paper for a potential $U$ describing forms only can now be applied to the extended version including the curvature terms. The relevant information is encoded in the vectors $\mathbf{q}$ which we have specified for forms in eq. (6), (7) and for curvature terms in eq. (99) above.

For example, as in the case of flat subspaces, a model is soluble if it corresponds to a Toda model. To check this, one has to compute the scalar products between all the vectors $\mathbf{q}$ now also including those describing curvature in analogy to eq. (61). This is easily done given the above expression (99). For instance, the scalar product between a vector $\mathbf{q}_{k}$ describing curvature in the subspace $k$ and a vector $\mathbf{q}^{(\mathrm{el})}$ for an elementary form is given by

$$
<\mathbf{q}_{k}, \mathbf{q}^{(\mathrm{el})}>=-2 \epsilon_{k},
$$

where we have used eq. (21), (22) and eq. (6). Recall, that $\epsilon_{k}=1$ if the subspace $k$ is occupied by the form and $\epsilon_{k}=0$ otherwise. Therefore, a model with one elementary form and one curved transverse subspace has orthogonal $\mathbf{q}$ vectors and can be solved following the lines of section 4.1. More generally, if a Toda model with curvature has been found by calculating all scalar products, it can be solved following the general procedure which we have described in section 4 .

By way of a specific example with a non-flat subspace, let us consider the cosmology with nontrivial NS three-form given by Behrndt and Förste [16]. Note that, unlike all previous examples, this is in five not ten dimensions. The solution is derived directly from the five-dimensional black hole solution with a NS three-form [24] by going to a regime where the time and radial coordinates exchange roles. The metric is given by

$$
d s^{2}=-\frac{d t^{2}}{\left(-1+t_{+}^{2} / t^{2}\right)\left(1-t_{-}^{2} / t^{2}\right)}+\frac{-1+t_{+}^{2} / t^{2}}{1-t_{-}^{2} / t^{2}} d y^{2}+t^{2} d \Omega^{2}
$$

where $d \Omega^{2}$ is the metric on a three-sphere, while the dilaton and the NS form are given by

$$
\phi=-\frac{1}{2} \ln \left(1-t_{-}^{2} / t^{2}\right)+\phi_{0} \quad H_{\mu_{1} \mu_{2} \mu_{3}}=2 t_{+} t_{-} t^{-6} \epsilon_{\mu_{1} \mu_{2} \mu_{3}}
$$

where $\mu_{1}, \mu_{2}$ and $\mu_{3}$ are coordinates on the three-sphere. We see immediately that this solution is in the form of our general Ansatz. Comparing with (96), we see that the space has been split into two subspaces: a one-dimensional subspace parameterized by y and a three-dimensional spherical subspace which together with the time direction constitutes our observed spacetime. Thus we would write $d_{0}=1$ and $d_{1}=3$ while $K_{0}=0$ and $K_{1}=1$. The NS form field spans only the three-sphere, without a component in the time direction. This implies a solitonic Ansatz for $H_{\mu_{1} \mu_{2} \mu_{3}}$. Using eqs. (18) and (99), we can immediately write down the vectors $\mathbf{q}_{H}$ and $\mathbf{q}_{K}$ corresponding to the NS field and the non-zero subspace curvature,

$$
\mathbf{q}_{H}=(2,0,8 / 7) \quad \mathbf{q}_{K}=(2,4,0) .
$$

If $u_{H}$ is the charge of the NS field, then, by the discussion above, the potential $U$ for this Ansatz is given by

$$
U=\frac{1}{2} u_{H}^{2} e^{\mathbf{q}_{H} \cdot \boldsymbol{\alpha}}-2 e^{\mathbf{q}_{K} \cdot \boldsymbol{\alpha}} .
$$


Similarly, we can calculate the metric on moduli space and its inverse for this five-dimensional Ansatz using eqs. (12) and (22). For the inverse metric we find

$$
G^{-1}=\left(\begin{array}{ccc}
\frac{1}{3} & -\frac{1}{6} & 0 \\
-\frac{1}{6} & 0 & 0 \\
0 & 0 & \frac{3}{8}
\end{array}\right) .
$$

This allows us to compute the scalar product between $\mathbf{q}_{H}$ and $\mathbf{q}_{K}$. We find that

$$
<\mathbf{q}_{H}, \mathbf{q}_{K}>=0
$$

so that, in fact, we have a model with orthogonal $\mathbf{q}$ vectors. As a result, it corresponds to a simple SU(2) Toda model and we can solve the equations of motion exactly following the discussion given in section 4.1. Choosing the correct time parameterization, we thus re-derive the solution of Behrndt and Förste given above. We note that there are many other models with a spherical subspace which could be solved in this way. It would be interesting to know if these can all be directly related to black $p$-brane solutions.

It is also interesting to note that the presence of a curved subspace significantly alters the form of the solution. We note that, unlike the previous cases, with a spherical subspace the corresponding term in the potential $U$ is negative. The effect, in a solution such as that of Behrndt and Förste, is that there is no longer necessarily either an initial or a final singularity. From the form of the metric in eq. (101), it would appear that the solution is divergent at $t=t_{+}$and $t=t_{-}$. However, in fact, the scalar curvature is finite at each point (essentially since they correspond to the two horizons of the origin black $p$-brane solution). This raises the very interesting possibility that solutions of this form may resolve the graceful exit problem inherent in the cosmological solutions given thus far, providing an inflating solution which does not end in a curvature singularity.

\section{Conclusion}

Throughout the paper we have noted the close relation between the cosmological solutions we have been discussing and supergravity $p$-brane solutions. The relationship consists of exchanging the time coordinate for a transverse spatial coordinate, thus transforming a cosmological space where everything evolves in time into a brane world-volume where the fields all depend on the transverse radial coordinate, and vice versa. This relationship is very suggestive. In particular, it raises the possibility that the statistical mechanics of Hawking radiation in cosmological spacetimes (which is responsible for large scale structure) could have a similar string theoretical origin as the entropy of black holes [7] (which can be computed from the associated D-branes). We are studying this possibility and will report on it elsewhere. 
Note: Shortly after this paper was finished, an interesting paper by Larsen and Wilczek [34] appeared which emphasizes that the solutions of Behrndt et. al. [16], Poppe et. al. [20] and some of those discussed in this paper actually exactly correspond to the interior of certain p-brane black holes, and gave several new cosmological solutions in this context. They independently point out that these solutions might evade the problem of curvature singularities.

Acknowledgments: We would like to thank F. Larsen and F. Wilczek for discussions. A. L. is supported by a fellowship from Deutsche Forschungsgemeinschaft (DFG). A. L. and B. A. O. are supported in part by DOE under contract No. DE-AC02-76-ER-03071. D. W. is supported in part by DOE under contract No. DE-FG02-91ER40671.

\section{References}

[1] R. Brustein and P. J. Steinhardt, Phys. Lett. B302 (1993) 196.

[2] T. Banks, M. Berkooz, S.H. Shenker, G. Moore and P.J. Steinhardt, Phys.Rev. D52 (1995) 3548 .

[3] C.M. Hull and P.K. Townsend, Nucl.Phys. B438 (1995) 109;

E. Witten, Nucl. Phys. B443 (1995) 85.

[4] J. Polchinski, Phys. Rev. Lett. 75 (1995) 4724;

J. Polchinski, S. Chaudhuri, C. V. Johnson, Notes on D-branes, NSF-ITP-96-003, hepth/9602052.

[5] For a review see M. J. Duff, R. R. Khuri and J. X. Lu, Phys. Rep. 259 (1995) 213.

[6] T. Banks and M. Dine, Couplings and scales in strongly coupled heterotic string theory, RU96-27, hep-th/9605136;

T. Banks and M. Dine, The cosmology of string theoretic axions, SCIPP-96-31, hep-th/9608197.

[7] A. Strominger and C. Vafa, Phys. Lett. B379 (1996) 99;

C. G. Callan, Jr. and J. M. Maldacena, Nucl. Phys. B472 (1996) 591.

[8] A. Lukas, B. A. Ovrut and D. Waldram, Cosmological Solutions of Type II String Theory, UPR-711T, hep-th/9608195, to appear in Phys. Lett. B.

[9] M. Mueller, Nucl. Phys. B337 (1990) 37.

[10] E. Kasner, Am. J. Math. 48 (1921) 217;

A. Chodos and S. Detweiler, Phys. Rev. D21 (1980) 2167. 
[11] G.W. Gibbons and P.K. Townsend, Nucl. Phys. B282 (1987) 610.

[12] P. G. O. Freund and M. Rubin, Phys. Lett. B97 (1980) 22.

[13] P. G. O. Freund, Nucl. Phys. B209 (1982) 146.

[14] I. Antoniadis, C. Bachas, J. Ellis, and D. V. Nanopoulos, Phys. Lett. B211 (1988) 393;

I. Antoniadis, C. Bachas, J. Ellis and D. V. Nanopoulos, Nucl. Phys. B328 (1989) 117.

[15] A. A. Tseytlin, Int. J. Mod. Phys. D1 (1992) 223.

[16] K.Behrndt and S. Förste, Nucl. Phys. B340 441.

[17] E. J. Copeland, A. Lahiri and D. Wands, Phys. Rev. D50 (1994) 4868;

R. Easther, K. Maeda and D. Wands, Phys. Rev. D53 (1996) 4247;

S. Mukherji, A note on Brans-Dicke cosmology with axion, CTP-TAMU-44-96, hepth/9609048.

[18] N. Kaloper, Stringy Toda cosmologies, MCGILL-96-34, hep-th/9609087, submitted to Phys. Rev. D

[19] H. Lü, S. Mukherji, C.N. Pope and K.W. Xu, Cosmological solutions in string theories CTPTAMU-51-96, hep-th/9610107.

[20] R. Poppe and S. Schwager, String Kaluza-Klein cosmologies with RR-fields, LMU-TPW 96-27, hep-th/9610166.

[21] M. Gasperini and G. Veneziano, Astropart. Phys. 1 (1993) 317; R. Brustein and G. Veneziano, Phys. Lett. B329 (1994) 429.

[22] See for instance the first reference of [23].

[23] G. Papadopoulos and P. K. Townsend, Nucl. Phys. B475 (1996) 149;

I. R. Klebanov and A. A. Tseytlin, Nucl. Phys. B475 (1996) 179;

J. P. Gauntlett, D. A. Kastor and J. Traschen Overlapping branes in M-theory, CALT-68-2055, hep-th/9604179;

N. Khviengia, Z. Khviengia, H. Lü and C. N. Pope, Intersecting M-branes and bound states, CTP-TAMU-19-96, hep-th/9605077;

H. Lü, C. N. Pope and K. S. Stelle, Vertical versus diagonal dimensional reduction for p-branes, CTP-TAMU-18-96, hep-th/9605082.

[24] G. W. Gibbons and K. Maeda, Nucl. Phys. B298 (1988) 741;

G. T. Horowitz and A. Strominger, Nucl. Phys. B360 (1991) 197;

M. J. Duff and J. X. Lu, Nucl. Phys. B416 (1994) 301. 
[25] H. Lü, C. N. Pope and K. W. Xu, Liouville and Toda Solitons in M-theory, CTP-TAMU12/96, hep-th/9604058; H. Lü and C. N. Pope, $S L(N+1, R)$ Toda Solitons in Supergravities, CTP-TAMU-24/96, hep-th/9607027.

[26] L. J. Romans, Phys. Lett. B169 (1986) 374.

[27] E. Bergshoeff, M. de Roo, M. B. Green, G. Papadopoulos and P. K. Townsend, Nucl. Phys. B470 (1996) 113.

[28] A. Lukas, B. A. Ovrut and D. Waldram Stabilizing Dilaton and Moduli Vacua in String and M-Theory Cosmology, UPR-726T, IASSNS-HEP-96/118, PUBT-1667, hep-th/9611204.

[29] C. Kounnas and D. Lüst, Phys. Lett. B289 (1992) 56.

[30] S. J. Rey, Back Reaction and Graceful Exit in String Inflationary Cosmology, SNUTP 96/043, hep-th/9605176.

[31] B. Kostant, Adv. Math. 34 (1979) 195.

[32] V. R. Gavrilov, V. D. Ivashchuk and V. N. Melnikov, Multidimensional Cosmology with Multicomponent Perfect Fluid and Toda Lattice, RGA-CSVR-009/94, gr-qc/9407019; Multidimensional Integrable Vacuum Cosmology with two Curvatures, RGA-CSVR-001/96, grqc/9602063.

[33] For Lie group properties and a list of Cartan matrices see for example R. Slansky, Phys. Rep. 73 (1981)

[34] F. Larsen and F. Wilczek, Resolution of Cosmological Singularities, IASSNS-HEP 96/108, hep-th/9610252. 